\title{
Entanglement of positive definite functions on compact groups
}

\author{
J. K. Korbicz ${ }^{1,2 *}$, J. Wehr ${ }^{3,2}$, and M. Lewenstein ${ }^{2}$ \\ ${ }^{1}$ Dept. d'Estructura i Constituents de la Matèria, Universitat de Barcelona, 647 Diagonal, \\ 08028 Barcelona, Spain \\ ${ }^{2}$ ICREA and ICFO-Institut de Ciències Fotòniques, Mediterranean Technology Park, 08860 \\ Castelldefels (Barcelona), Spain \\ ${ }^{3}$ Department of Mathematics, University of Arizona, 617 N. Santa Rita Ave., Tucson, AZ \\ 85721-0089, USA
}

\begin{abstract}
We define and study entanglement of continuous positive definite functions on products of compact groups. We formulate and prove an infinite-dimensional analog of the Horodecki Theorem, giving a necessary and sufficient criterion for separability of such functions. The resulting characterisation is given in terms of mappings of the space of continuous functions, preserving positive definiteness. A relation between the developed group-theoretical formalism and the conventional one, given in terms of density matrices, is established through the non-commutative Fourier analysis. It shows that the presented method plays the role of a "generating function" formalism for the theory of entanglement.
\end{abstract}

\section{Introduction}

Entanglement is a property of states of composite quantum mechanical systems. This concept lies at the very heart of quantum mechanics, and it concerns all of the important aspects of quantum theory: from philosophical aspects [1, 2, through physical [3] and mathematical [4, 5] fundamental 1 , to applications in quantum information and metrology [7]. The importance of entangled states for the understanding of quantum theory was recognized quite early, mainly thanks to Einstein (e.g. in the famous EPR paper by Einstein, Podolsky, and Rosen [8]). Only with the advent of new experimental techniques in recent years, it became clear that entanglement may in fact also be used as a resource for transmission and processing of (quantum) information, e.g. for quantum cryptography or quantum computing (for a recent review, see Ref. [4] ; see also Ref. [7]).

In the present work we develop a novel framework for studying quantum entanglement, based on analysis of continuous functions on compact groups. With respect to the standard formalism of entanglement theory, our approach plays a role analogous to that of a "generating function" method - various group-theoretical objects serve as "generating functions" for the corresponding families of operator-algebraic objects (like density matrices, positive maps, etc), operating in different dimensions. This allows one to formulate and address the questions of entanglement theory in a unified, dimension-wise, way.

Before we proceed with the group-theoretical formalism, let us first recall some basic facts and define the notion of entanglement precisely. A quantum system is associated with a Hilbert space $\mathcal{H}$, which we will assume to have a countable basis. A state of the system is then represented by a positive, trace-class operator $\varrho$ (a density matrix), satisfying normalization condition $\operatorname{tr} \varrho=1$. If the system under consideration is composite, i.e. it can be thought of being composed of two subsystems $\mathscr{A}$ and $\mathscr{B}$, each of which is treated as an independent

\footnotetext{
*jaroslaw.korbicz@icfo.es

${ }^{1}$ For a description of positive maps from a physical point of view, see e.g. Ref. [6].
} 
individual, then, according to the postulates of quantum theory, the Hilbert space of the system is $\mathcal{H}=\mathcal{H}_{\mathscr{A}} \otimes \mathcal{H}_{\mathscr{B}}$. The following definition thus makes sense [9]:

Definition 1.1 A state $\varrho$ on $\mathcal{H}_{\mathscr{A}} \otimes \mathcal{H}_{\mathscr{B}}$ is called separable if it can be approximated in the trace norm by convex combinations of the form:

$$
\sum_{m=1}^{K} p_{m}\left|x_{m}\right\rangle\left\langle x_{m}|\otimes| y_{m}\right\rangle\left\langle y_{m}\right|, \quad \text { where } x_{m} \in \mathcal{H}_{\mathscr{A}}, y_{m} \in \mathcal{H}_{\mathscr{B}}, p_{m} \geqslant 0, \sum_{m=1}^{K} p_{m}=1 .
$$

Otherwise @ is called entangled.

This definition can be easily generalized to multipartite systems with more than two parties involved.

In the light of the Definition 1.1 a natural question arises, known as the separability problem: Given a state $\varrho$ decide if it is separable or not.

The problem turns out to be computationally very hard: although efficient algorithms employing positive definite programming methods exist in lower dimensions [10] (for a specific formulation of semi-definite approach for $2 \otimes N$ systems see Ref. [11), it has been proven that the problem belongs to the $N P$ complexity class as dimensions of the Hilbert spaces involved grow [12]. In term of operational entanglement criteria up to date there are only partial answers known, in both finite and infinite dimensions. We briefly quote below few basic results, referring the reader to Ref. [4 for a complete overview. One astonishingly powerful, given its simplicity, necessary criterion for separability follows immediately from the definition of separable states [13, 14]:

Theorem 1.1 (Positivity of Partial Transpose (PPT)) If a state $\varrho$ on $\mathcal{H}_{\mathscr{A}} \otimes \mathcal{H}_{\mathscr{B}}$ is separable then the partially transposed operator $\varrho^{T_{\mathscr{B}}}:=\left(\mathbf{1}_{\mathscr{A}} \otimes T\right) \varrho$ is positive, where $T$ is a transposition map and $\mathbf{1}_{\mathscr{A}}$ is the identity operator on $\mathcal{H}_{\mathscr{A}}$.

In the lowest non-trivial dimensions $\operatorname{dim} \mathcal{H}_{\mathscr{A}}=\operatorname{dim} \mathcal{H}_{\mathscr{B}}=2$ and $\operatorname{dim} \mathcal{H}_{\mathscr{A}}=2, \operatorname{dim} \mathcal{H}_{\mathscr{A}}=3$ PPT criterion provides both necessary and sufficient condition for separability (see Ref. [14, Theorem 3). However, in higher dimensions there exist states, called PPT or bound entangled, which satisfy the PPT criterion, but are nevertheless entangled. The first examples of such states were constructed in Ref. [15] (although in a different context of, so called, indecomposable maps) and in Ref. [16].

In infinite dimension, a complete solution to the separability problem exists only for a special family of states - so called Gaussian states [17.

As mentioned above the separability problem is connected to other open mathematical problems. In their fundamental work [14 Horodecki et al. established an important link between this problem and the problem of characterization of positive maps on finite-dimensional matrix algebras (cf. Ref. [14], Theorem 2; see also Refs. [5, 15, 18, 19] ):

Theorem 1.2 (M., P., and R. Horodecki) Let $\mathcal{L}(\mathcal{H})$ denote the space of linear operators on $\mathcal{H}$ and let $\varrho \in \mathcal{L}\left(\mathcal{H}_{\mathscr{A}} \otimes \mathcal{H}_{\mathscr{B}}\right)$ be a density matrix on a finite dimensional Hilbert space $\mathcal{H}_{\mathscr{A}} \otimes \mathcal{H}_{\mathscr{B}} . \quad$ Matrix $\varrho$ is separable if and only if for all linear maps $\Phi: \mathcal{L}\left(\mathcal{H}_{\mathscr{B}}\right) \rightarrow \mathcal{L}\left(\mathcal{H}_{\mathscr{A}}\right)$ preserving positive operators (such maps are called positive), $\left(\mathbf{1}_{\mathscr{A}} \otimes \Phi\right) \varrho \geqslant 0$ as an operator on $\mathcal{H}_{\mathscr{A}} \otimes \mathcal{H}_{\mathscr{A}}$. 
The starting point for the present work is the non-commutative Fourier analysis on a compact group, which we proposed to employ for studying entanglement in Ref. [20] (cf. Ref. [21] where the same method was used for a rigorous derivation of the classical limit of quantum state space). Namely, one can pass from operators $A \in \mathcal{L}\left(\mathcal{H}_{\mathscr{A}} \otimes \mathcal{H}_{\mathscr{B}}\right)$ to their non-commutative Fourier transforms 2 in two steps: i) identify the spaces $\mathcal{H}_{\mathscr{A}}, \mathcal{H}_{\mathscr{B}}$ with representation spaces of unitary, irreducible representations $\pi_{\alpha}, \tau_{\beta}$ of some compact groups $G_{1}$ and $G_{2}$ respectively (there are no a priori restrictions on $G_{1}, G_{2}$ apart from possessing representations in suitable dimensions); ii) pass from $A$ to a function $\varphi_{A}: G_{1} \times G_{2} \rightarrow \mathbb{C}$, the non-commutative Fourier transform of $A$, through:

$$
A \mapsto \varphi_{A}\left(g_{1}, g_{2}\right):=\operatorname{tr}\left[A \pi_{\alpha}\left(g_{1}\right) \otimes \tau_{\beta}\left(g_{2}\right)\right] .
$$

The above transform is called non-commutative, since apart from the trivial case $\operatorname{dim} \mathcal{H}_{\mathscr{A}}=$ $\operatorname{dim} \mathcal{H}_{\mathscr{B}}=1$, groups $G_{1}$ and $G_{2}$ are necessarily non-Abelian. In case $A=\varrho$ is a quantum state, the corresponding function $\varphi_{\varrho}$ is called non-commutative characteristic function of $\varrho$. The transformation (2) is invertible-one can recover $A$ from $\varphi_{A}$. Hence, one expects that for density matrices their non-commutative characteristic functions should encode entanglement in some way [20]. This is indeed the case and in what follows we define and study the notion of separability for suitably generalized non-commutative characteristic functions (general continuous positive definite functions on $G_{1} \times G_{2}$; cf. Definition 2.1). We then prove an analog of the Horodecki Theorem 1.2 for such functions, which constitutes the main result of the paper. Since the framework we work in is countably infinite-dimensional (unless both $G_{1}, G_{2}$ are finite) our result can be viewed as a generalization of Horodecki Theorem to an infinite-dimensional setting. The usual quantum-mechanical formalism, given by density matrices, and the presented group-theoretical one are then shown to be related through non-commutative harmonic analysis. In particular, by employing non-commutative Fourier transform we demonstrate how our approach turns out to be a "generating function" method for the theory of entanglement.

Let us finally remark that the formalism of non-commutative Fourier transform (2) is closely related to that of generalized coherent states [23. The difference is that in the coherent state formalism one assigns to an operator $A$ a function (called $P$-representation of $A$ ), which is defined not on the whole group $G$, but on a homogeneous space $G / H$, where $H$ is an isotropy subgroup of a fixed vector. However, unlike non-commutative Fourier transform $\varphi_{A}, P$-representation is generally non-unique (e.g. in $S U(2)$ case) and does not encode positivity of a density matrix in a simple manner. For some applications of generalized coherent states to the study of entanglement see e.g. Refs. [24].

\section{Preliminary notions}

In the main part of the work $G_{1}, G_{2}$ will be compact groups. The principal object of our study are continuous positive definite functions on the product group $G_{1} \times G_{2}$. But first we recall some basic definitions and facts, valid for any locally compact $G$ (see e.g. Refs. [25, 26, 27] for a complete exposition).

\footnotetext{
${ }^{2}$ We note that in Ref. [22] the term "noncommutative Fourier transform" is used in a slightly differentthough very closely related-sense.
} 
Definition 2.1 A continuous complex function $\varphi$ on a group $G$ with the Haar measure $d g$ is called positive definite if it is bounded and satisfies:

$$
\iint d g d h \overline{f(g)} \varphi\left(g^{-1} h\right) f(h) \geqslant 0
$$

(bar denotes complex conjugation) for any continuous function $f$ with compact support.

We will denote by $\mathcal{P}(G)$ the set of positive definite functions on $G$ and by $\mathcal{P}_{1}(G)$ its subset consisting of the functions which satisfy the normalization $\varphi(e)=1$, where $e$ is the neutral element of $G \cdot \mathcal{P}(G)$ is a closed convex cone in $C(G)$ - the space of continuous complex-valued functions on $G$ equipped with the topology of uniform convergence on compact sets, called compact convergence in the sequel.

The structure of $\mathcal{P}(G)$ is described by the following deep, fundamental result of representation theory, often referred to as the GNS construction (see e.g. Ref. [25], Theorem 3.20; Ref. [26], Theorem 13.4.5):

Theorem 2.1 (Gel'fand, Naimark, Segal) With every $\varphi \in \mathcal{P}(G)$ we can associate a Hilbert space $\mathcal{H}_{\varphi}$, a unitary representation $\pi_{\varphi}$ of $G$ in $\mathcal{H}_{\varphi}$ and a vector $v_{\varphi}$, cyclic for $\pi_{\varphi}$, such that:

$$
\varphi(g)=\left\langle v_{\varphi} \mid \pi_{\varphi}(g) v_{\varphi}\right\rangle .
$$

The representation $\pi_{\varphi}$ is unique up to a unitary equivalence.

The above result provides a tool for a systematic study of $\mathcal{P}(G)$ in terms of representations of $G$ (and conversely). In the sequel we will need some basic properties of positive-definite functions. While they all follow from the definition by standard, elementary arguments, we find the proofs based on the GNS representation particularly transparent.

A function $\varphi$ will be called pure if $\pi_{\varphi}$ is irreducible. Pure normalized functions are the extreme points of $\mathcal{P}_{1}(G)$ (cf. Ref. [25], Theorem 3.25); we denote their set by $\mathcal{E}_{1}(G)$. Every $\varphi \in \mathcal{P}_{1}(G)$ is a limit, in the topology of compact convergence, of convex combinations of extreme points of $\mathcal{P}_{1}(G)$ (cf. Ref. [26], Theorem 13.6.4):

$$
g \mapsto \sum_{m=1}^{N} p_{m} \varepsilon_{m}(g), \text { where } \varepsilon_{m} \in \mathcal{E}_{1}(G), p_{m} \geqslant 0, \sum_{m=1}^{N} p_{m}=1 .
$$

There is also an integral representation (provided $G$ is separable as a topological space), sometimes called generalized Bochner Theorem. Namely, for any $\varphi \in \mathcal{P}_{1}(G)$ there exists a probability measure $\mu_{\varphi}$ concentrated on $\mathcal{E}_{1}(G)$ such that (cf. Ref. [26], Proposition 13.6.8):

$$
\varphi(g)=\int_{\mathcal{E}_{1}(G)} d \mu_{\varphi}(\varepsilon) \varepsilon(g) \quad \text { for any } g \in G .
$$

From this point on, we assume that $G_{1}, G_{2}$ are compact and consider positive definite functions on $G_{1} \times G_{2}$. Let us introduce the algebraic tensor product $C\left(G_{1}\right) \otimes C\left(G_{2}\right)$ as the space of finite (complex) linear combinations of product functions $f \otimes \xi:\left(g_{1}, g_{2}\right) \mapsto f\left(g_{1}\right) \xi\left(g_{2}\right)$. Then $C\left(G_{1}\right) \otimes C\left(G_{2}\right)$ is uniformly dense in $C\left(G_{1} \times G_{2}\right)$. This standard fact follows, for example, from the Stone-Weierstrass Theorem (see e.g. Ref. [28]). Every product $\phi \otimes \psi$, where 
$\phi \in \mathcal{P}_{1}\left(G_{1}\right), \psi \in \mathcal{P}_{1}\left(G_{2}\right)$, is positive definite on $G_{1} \times G_{2}$, since, by the GNS Theorem 2.1, $\phi\left(g_{1}\right) \psi\left(g_{2}\right)=\left\langle v_{\phi} \otimes v_{\psi} \mid \pi_{\phi}\left(g_{1}\right) \otimes \pi_{\psi}\left(g_{2}\right) v_{\phi} \otimes v_{\psi}\right\rangle$ which is of the form (4) on $G_{1} \times G_{2}$. It follows that convex combinations of such products are positive definite and hence so are uniform limits of such convex combinations. The resulting class of positive definite functions, introduced formally in the next definition, is our fundamental object of study (compare Definition 1.1).

Definition 2.2 We define Sep $p_{0}$ as the set of all functions $\varphi \in \mathcal{P}_{1}\left(G_{1} \times G_{2}\right)$ which can be represented as finite convex combinations

$$
\varphi\left(g_{1}, g_{2}\right)=\sum_{m=1}^{K} p_{m} \varepsilon_{m}\left(g_{1}\right) \eta_{m}\left(g_{2}\right), \text { where } \varepsilon_{m} \in \mathcal{E}_{1}\left(G_{1}\right), \eta_{m} \in \mathcal{E}_{1}\left(G_{2}\right) .
$$

A function $\varphi \in \mathcal{P}_{1}\left(G_{1} \times G_{2}\right)$ is called separable if it is a uniform limit of elements of Sep 0 . The set of separable functions is denoted by Sep. Functions which are not separable are called entangled.

The definitions of separable and entangled functions generalize without any change to arbitrary (i.e. not necessarily normalized) positive definite functions. This includes our main result, Theorem 3.2, together with its proof (since for a nonzero positive definite function $\varphi\left(e_{1}, e_{2}\right)=\|\varphi\|_{\infty}>0$, we can replace $\varphi$ by $\varphi / \varphi\left(e_{1}, e_{2}\right)$ and reduce the proof to the normalized case). The normalization is, however, natural from the physical point of view.

Geometrically $\mathcal{E}_{1}\left(G_{1}\right) \times \mathcal{E}_{1}\left(G_{2}\right)$ is embedded into $\mathcal{E}_{1}\left(G_{1} \times G_{2}\right)$ through the map $(\varepsilon, \eta) \mapsto \varepsilon \otimes \eta$. Then $S e p$ is a closed convex hull of $\mathcal{E}_{1}\left(G_{1}\right) \times \mathcal{E}_{1}\left(G_{2}\right)$.

We note that every $\varphi \in S e p$ admits an integral representation:

$$
\varphi\left(g_{1}, g_{2}\right)=\int_{\mathcal{E}_{1}\left(G_{1}\right) \times \mathcal{E}_{1}\left(G_{2}\right)} d \mu_{\varphi}(\varepsilon, \eta) \varepsilon\left(g_{1}\right) \eta\left(g_{2}\right) \quad \text { for any }\left(g_{1}, g_{2}\right) \in G_{1} \times G_{2},
$$

but we will not use this fact.

\section{Necessary and sufficient criterion for separability of pos- itive definite functions}

The main problem we would like to address is that of finding an intrinsic characterization of separable functions $\varphi \in$ Sep. This is known as the generalized separability problem [20]. By Eq. (44) for every positive definite function $\phi, \phi\left(g^{-1}\right)=\overline{\phi(g)}$ and this function is again positive definite. It now follows immediately from Definition 2.2 and from uniform closedness of $\mathcal{P}\left(G_{1} \times G_{2}\right)$ in $C\left(G_{1} \times G_{2}\right)$ that:

Theorem 3.1 If $\varphi \in$ Sep then the function $\left(g_{1}, g_{2}\right) \mapsto \varphi\left(g_{1}, g_{2}^{-1}\right)$ is positive definite.

The above simple criterion is only a necessary condition - there are functions satisfying it which are nevertheless entangled. This can be seen by noting that Theorem 3.1 is a grouptheoretical analog of the PPT criterion, given by Theorem 1.1, (we will show it in Section 5, see also Ref. [20], Theorem 2) and, as we mentioned in the Introduction, there exist PPT entangled (or, equivalently, bound entangled) quantum states [16]. A natural question arises whether one obtains a complete characterization of separable functions when in place of the inverse $g \mapsto g^{-1}$ one considers all possible linear maps of functions, preserving positive definiteness. The affirmative answer is the main result of our work: 
Theorem 3.2 A function $\varphi \in \mathcal{P}_{1}\left(G_{1} \times G_{2}\right)$ is separable if and only if for every bounded linear map $\Lambda: C\left(G_{2}\right) \rightarrow C\left(G_{1}\right)$, such that $\Lambda \mathcal{P}\left(G_{2}\right) \subset \mathcal{P}\left(G_{1}\right)$, function $(i d \otimes \Lambda) \varphi$ is positive definite on $G_{1} \times G_{1}$.

Tensor product id $\otimes \Lambda: C\left(G_{1} \times G_{2}\right) \rightarrow C\left(G_{1} \times G_{1}\right)$ is defined in the natural way: we first define it on the algebraic product $C\left(G_{1}\right) \otimes C\left(G_{2}\right)$ and then extend by continuity to all of $C\left(G_{1} \times G_{2}\right)$.

The above theorem is a group-theoretical analog of the Horodecki Theorem 1.2. In fact, we derive a version of the Horodecki result as a corollary in Section 4 (cf. Theorem 4.2). We will adopt the standard terminology of entanglement theory and say that an entangled function $\varphi$ is detected by a map $\Lambda$ if the function (id $\otimes \Lambda$ ) $\varphi$ is not positive definite.

As mentioned earlier, the above theorem, as well as the following proof, hold for arbitrary positive definite functions, but in order to use more natural concepts from the physical point of view we state and prove it for normalized ones. The proof in one direction is immediate and follows directly from the Definition 2.2] (id $\otimes \Lambda) \sum_{m=1}^{K} p_{m} \varepsilon_{m} \otimes \eta_{m}=\sum_{m=1}^{K} p_{m} \varepsilon_{m} \otimes \Lambda \eta_{m} \in$ $\mathcal{P}\left(G_{1} \times G_{1}\right)$ and since $\mathcal{P}\left(G_{1} \times G_{1}\right)$ is uniformly closed in $C\left(G_{1} \times G_{1}\right)$ this holds on all of Sep.

For the proof in the other direction, let $C\left(G_{1} \times G_{2}\right)^{\prime}$ denote the space of continuous linear functionals on $C\left(G_{1} \times G_{2}\right)$ (the space dual to $\left.C\left(G_{1} \times G_{2}\right)\right)$. Since $S e p$ is a closed convex set, it follows from the Hahn-Banach Theorem (see e.g. Ref. [29], Theorem V.4) that for every $\varphi \notin S e p$ there exists a functional $l \in C\left(G_{1} \times G_{2}\right)^{\prime}$ and a real number $\gamma$, such that:

$$
\operatorname{Rel}(\varphi)<\gamma \leqslant \operatorname{Rel}(\sigma) \text { for any } \sigma \in \operatorname{Sep},
$$

where $\operatorname{Rel}$ denotes the real part of the functional $l: \operatorname{Re} l(\varphi):=\operatorname{Re}[l(\varphi)]$. From the Riesz Representation Theorem (see e.g Ref. [29], Theorem IV.17) we know that each linear functional $l$ on $C\left(G_{1} \times G_{2}\right)$ can be uniquely represented by a complex measure $\mu_{l}$ with finite total variation $\left|\mu_{l}\right|$ on $G_{1} \times G_{2}$. Denoting the space of such measures by $M\left(G_{1} \times G_{2}\right)$ we have: $C\left(G_{1} \times G_{2}\right)^{\prime}=$ $M\left(G_{1} \times G_{2}\right)$. We will interchangeably treat elements of $C\left(G_{1} \times G_{2}\right)^{\prime}$ as either linear functionals or as the corresponding measures. To work with the normalized functions $\varphi$ which we are interested in, it is convenient to introduce a modification of the functional $l$ in (9): $L:=l-\gamma \delta_{\left(e_{1}, e_{2}\right)}$, where $\delta_{\left(e_{1}, e_{2}\right)}$ is the Dirac delta (point mass), concentrated at the neutral element $\left(e_{1}, e_{2}\right)$ of $G_{1} \times G_{2}$. Hence, for every entangled $\varphi \in \mathcal{P}_{1}\left(G_{1} \times G_{2}\right)$ there exists $L \in C\left(G_{1} \times G_{2}\right)^{\prime}$ such that:

$$
\operatorname{Re} L(\varphi)<0 \leqslant \operatorname{Re} L(\sigma) \text { for any } \sigma \in \operatorname{Sep} .
$$

As an easy consequence of the above condition we obtain the following lemma, crucial for the rest of the proof:

Lemma 3.1 A function $\varphi$ is separable if and only if for every functional $L \in C\left(G_{1} \times G_{2}\right)^{\prime}$, satisfying $\operatorname{Re} L\left(\psi_{1} \otimes \psi_{2}\right) \geqslant 0$ for every $\psi_{1} \in \mathcal{P}_{1}\left(G_{1}\right), \psi_{2} \in \mathcal{P}_{1}\left(G_{2}\right)$, we have $\operatorname{Re} L(\varphi) \geqslant 0$.

Indeed, for every $\operatorname{Sep}_{0} \ni \varphi=\sum_{m=1}^{K} p_{m} \varepsilon_{m} \otimes \eta_{m}, L(\varphi)=\sum_{m=1}^{K} p_{m} L\left(\varepsilon_{m} \otimes \eta_{m}\right) \geqslant 0$ and by continuity of $L$ this extends to all of Sep. Conversely, assume that for every $L$ satisfying the condition in the statement of the lemma, $\operatorname{Re} L(\varphi) \geqslant 0$ but $\varphi \notin S e p$. Then from the HahnBanach Theorem (see 10) we know that there exists a functional $L_{0}$ such that $\operatorname{Re} L_{0}(\sigma) \geqslant 0$ for every separable $\sigma$-in particular for every function of the form $\psi_{1} \otimes \psi_{2}$-and $\operatorname{Re} L_{0}(\varphi)<0$, which contradicts our assumption. 
In order to pass from linear functionals on $C\left(G_{1} \times G_{2}\right)$ to linear maps from $C\left(G_{2}\right)$ to $C\left(G_{1}\right)$, we first employ an algebraic isomorphism between functionals from $C\left(G_{1} \times G_{2}\right)^{\prime}$ and bounded linear maps $\widetilde{\Lambda}: C\left(G_{2}\right) \rightarrow C\left(G_{1}\right)^{\prime}$. For each $L \in C\left(G_{1} \times G_{2}\right)^{\prime}$ we define the corresponding map $\widetilde{\Lambda}_{L}$ by:

$$
\widetilde{\Lambda}_{L} \xi(f):=L(f \otimes \xi)
$$

$\widetilde{\Lambda}_{L}$ is bounded:

$$
\left\|\widetilde{\Lambda}_{L}\right\|=\sup _{\|\xi\|_{\infty}=1}\left\|\widetilde{\Lambda}_{L} \xi\right\|_{\infty}^{\prime}=\sup _{\|\xi\|_{\infty}=1} \sup _{\|f\|_{\infty}=1}\left|\widetilde{\Lambda}_{L} \xi(f)\right|=\sup _{\|\xi\|_{\infty}=1} \sup _{\|f\|_{\infty}=1}|L(f \otimes \xi)|
$$

where $\|\cdot\|_{\infty}^{\prime}$ is the norm on $C\left(G_{1}\right)^{\prime}$ induced by the supremum norm $\|\cdot\|_{\infty}$. Conversely, for any bounded map $\widetilde{\Lambda}: C\left(G_{2}\right) \rightarrow C\left(G_{1}\right)^{\prime}$, Eq. (11) defines a functional $L_{\widetilde{\Lambda}}$ on $C\left(G_{1}\right) \otimes C\left(G_{2}\right)$, which is bounded by Eq. (12), and uniquely defined, since if $L_{\widetilde{\Lambda}}(f \otimes \xi)=0$ for all $f \in C\left(G_{1}\right), \xi \in$ $C\left(G_{2}\right)$, then by Eq. (11) $\widetilde{\Lambda} \equiv 0$ (note that $L_{\widetilde{\Lambda}}$ depends linearly on $\widetilde{\Lambda}$ ). As $C\left(G_{1}\right) \otimes C\left(G_{2}\right)$ is uniformly dense in $C\left(G_{1} \times G_{2}\right), L_{\widetilde{\Lambda}}$ can be uniquely extended to a continuous functional on all of $C\left(G_{1} \times G_{2}\right)$. This establishes the claimed isomorphism $L \leftrightarrow \widetilde{\Lambda}_{L}$.

Next, we establish a positivity criterion $\widetilde{\Lambda}_{L}$, analogous to the one given by Jamiołkowski in Ref. [30] for operators on finite dimensional Hilbert spaces:

Lemma 3.2 A functional $L \in C\left(G_{1} \times G_{2}\right)^{\prime}$ satisfies $\operatorname{Re} L\left(\psi_{1} \otimes \psi_{2}\right) \geqslant 0$ for all $\psi_{1} \in \mathcal{P}_{1}\left(G_{1}\right), \psi_{2} \in$ $\mathcal{P}_{1}\left(G_{2}\right)$ if and only if $R e \widetilde{\Lambda}_{L}$ maps positive definite functions from $\mathcal{P}\left(G_{2}\right)$ to positive definite measures from $M\left(G_{1}\right)$. (3) 3 :

Positive definite measure on $G$ is a measure $\mu$ satisfying a generalization of the condition

$$
\int d \mu\left(f^{*} * f\right) \geqslant 0 \text { for any } f \in C(G)
$$

where $f^{*}(g):=\overline{f\left(g^{-1}\right)}$ is the involution and $(f * \xi)(h):=\int d g f(g) \xi\left(g^{-1} h\right)$ is the convolution. The action of the map $\operatorname{Re} \widetilde{\Lambda}_{L}$ on $f$ is defined as the real part of the functional $\widetilde{\Lambda}_{L} f$. The condition $\operatorname{Re} L\left(\psi_{1} \otimes \psi_{2}\right) \geqslant 0$ for all normalized $\psi_{1} \in \mathcal{P}_{1}\left(G_{1}\right), \psi_{2} \in \mathcal{P}_{1}\left(G_{2}\right)$ is equivalent to $\operatorname{Re} L\left(\psi_{1} \otimes \psi_{2}\right) \geqslant 0$ for all $\psi_{1} \in \mathcal{P}\left(G_{1}\right), \psi_{2} \in \mathcal{P}\left(G_{2}\right)$, since we can replace $\psi_{1}, \psi_{2} \neq 0$ by $\psi_{1} / \psi_{1}(e)$ and $\psi_{2} / \psi_{2}(e)$. From the definition (11) it follows that:

$$
\operatorname{Re} L\left(\psi_{1} \otimes \psi_{2}\right)=\operatorname{Re}\left[\widetilde{\Lambda}_{L} \psi_{2}\left(\psi_{1}\right)\right]=\operatorname{Re} \widetilde{\Lambda}_{L} \psi_{2}\left(\psi_{1}\right)
$$

A theorem by Godement (cf. Ref. [31, Theorem 17; Ref. [26], Theorem 13.8.6) states that every positive definite function can be uniformly approximated by functions of the form $f^{*} * f$ where $f$ is continuous with compact support. Hence, since $G_{1}, G_{2}$ are compact, $\operatorname{Re} L\left(\psi_{1} \otimes \psi_{2}\right) \geqslant 0$ for every $\psi_{1} \in \mathcal{P}\left(G_{1}\right), \psi_{2} \in \mathcal{P}\left(G_{2}\right)$ if and only if $\operatorname{Re} L\left[\left(f^{*} * f\right) \otimes \psi_{2}\right]=\operatorname{Re} \widetilde{\Lambda}_{L} \psi_{2}\left(f^{*} * f\right) \geqslant 0$ for every $f \in C\left(G_{1}\right)$ and $\psi_{2} \in \mathcal{P}\left(G_{2}\right)$. But by Eqs. (13) and (14) this is equivalent to the measure $\operatorname{Re} \widetilde{\Lambda}_{L} \psi_{2} \in M\left(G_{1}\right)$ being positive definite for every $\psi_{2} \in \mathcal{P}\left(G_{2}\right)$.

\footnotetext{
${ }^{3}$ On an arbitrary locally compact $G$ positive definite measures are defined by requiring that condition (13) hold for all continuous functions with compact support (cf. Ref. [26], Definition 13.7.1).
} 
As the next step we will regularize maps $\widetilde{\Lambda}$. Note that for any $\mu \in M\left(G_{1}\right)$ and $f \in C\left(G_{1}\right)$ the convolution:

$$
\mu * f(h)=\int d \mu(g) f\left(g^{-1} h\right)
$$

is a continuous function on $G_{1}$. Let $\left\{\psi_{U}\right\}$, where $U \subset G_{1}$ runs through a neighbourhood base of the neutral element $e_{1} \in G_{1}$, be an approximate identity in $C\left(G_{1}\right)$. That is, for every $U$ we have:

$$
\begin{aligned}
& \text { o) } \left.\psi_{U} \in C\left(G_{1}\right), \quad i\right) \operatorname{supp} \psi_{U} \text { is a compact subset of } U \\
& \text { ii) } \left.\psi_{U} \geqslant 0, \quad \text { iii) } \psi_{U}\left(g^{-1}\right)=\psi_{U}(g), \quad i v\right) \int \psi_{U}=1
\end{aligned}
$$

Using definition (15), let us define for every $f \in C\left(G_{1}\right)$ functions

$$
\Lambda_{U} f:=\widetilde{\Lambda} f * \psi_{U}
$$

Then $\Lambda_{U} f$ converges in weak-* topology to $\widetilde{\Lambda} f$ as $U \rightarrow\left\{e_{1}\right\}$. To see this, let us calculate $\int d g \Lambda_{U} f(g) \xi(g)$ for an arbitrary $\xi \in C\left(G_{1}\right)$ :

$$
\begin{aligned}
\int d g\left(\widetilde{\Lambda} f * \psi_{U}\right)(g) \xi(g) & =\int d g \int d(\widetilde{\Lambda} f)(h) \psi_{U}\left(h^{-1} g\right) \xi(g)=\int d(\widetilde{\Lambda} f)(h) \int d g \psi_{U}\left(g^{-1} h\right) \xi(g) \\
& =\widetilde{\Lambda} f\left(\xi * \psi_{U}\right)
\end{aligned}
$$

where in the second step we used the symmetry of $\psi_{U}$ : $\psi_{U}\left(g^{-1}\right)=\psi_{U}(g)$. But from the properties (16), (17) of $\left\{\psi_{U}\right\}$ it follows that $\xi * \psi_{U} \rightarrow \xi$ uniformly as $U \rightarrow\left\{e_{1}\right\}$ (cf. Ref. 25], Theorem 2.42), which proves the desired weak-* convergence. Thus any bounded map $\widetilde{\Lambda}: C\left(G_{2}\right) \rightarrow M\left(G_{1}\right)$ can be weakly-* approximated by bounded maps $\Lambda_{U}: C\left(G_{2}\right) \rightarrow C\left(G_{1}\right)$ (boundedness of $\Lambda_{U}$ for every $U \subset G_{1}$ follows immediately from the definitions (15) and (18)) ).

In order to preserve the positivity property of the $\widetilde{\Lambda}$ 's, introduced in Lemma 3.2, we choose regularizing functions $\psi_{U}$ in a special way. Namely, for every neighbourhood $U$ of $e_{1} \in G_{1}$ we can find such open $V \ni e_{1}$ that: (cf. Ref. [25], Lemma 5.24):

$$
\text { o) } V \subset U, \quad \text { i) } V^{-1}=V, \text { ii) } g V g^{-1}=V \text { for every } g \in G_{1} \text {. }
$$

Let us define the functions:

$$
\begin{aligned}
\kappa_{V} & :=\frac{1}{|V|} \chi_{V} \\
\psi_{U} & :=\kappa_{V} * \kappa_{V},
\end{aligned}
$$

where $\chi_{V}$ is the indicator function of $V$ and $|V|=\int d g \chi_{V}(g)$ is the Haar measure of $V$. Then one easily shows that $\left\{\psi_{U}\right\}$ form an approximate identity in $C\left(G_{1}\right)$. Moreover, $\kappa_{V}$, and hence $\psi_{U}$, are central functions, i.e. for every $g$ and $h, \kappa_{V}(g h)=\kappa_{V}(h g)$, which follows from the property $(i i)$ of the sets $V$. Using functions (22) to regularize an arbitrary map $\widetilde{\Lambda}$, we find that:

$$
\Lambda_{U} f=\widetilde{\Lambda} f * \psi_{U}=\kappa_{V} * \widetilde{\Lambda} f * \kappa_{V}=\kappa_{V}^{*} * \widetilde{\Lambda} f * \kappa_{V}
$$

where in the second step we used the fact that $\kappa_{V}$ are central and hence $\mu * \kappa_{V}=\kappa_{V} * \mu$ for any $\mu$. In the last step we used the symmetry condition $(i)$ and $\overline{\chi_{V}}=\chi_{V}$. Now, from the special form of the regularization (23) we obtain: 
Lemma 3.3 If $\widetilde{\Lambda}: C\left(G_{2}\right) \rightarrow M\left(G_{1}\right)$ maps positive definite functions into positive definite measures, then for every neighbourhood $U$ of $e_{1} \in G_{1}$ the regularized maps $\Lambda_{U}$, defined by Eqs. (21), map $\mathcal{P}\left(G_{2}\right)$ into $\mathcal{P}\left(G_{1}\right)$.

To prove Lemma 3.3, note that for an arbitrary $\phi \in \mathcal{P}\left(G_{2}\right)$ and $f \in C\left(G_{1}\right)$ one has:

$$
\begin{aligned}
& \iint d g d h \overline{f(g)}\left(\kappa_{V}^{*} * \widetilde{\Lambda} \phi * \kappa_{V}\right)\left(g^{-1} h\right) f(h) \\
& =\iiint d g d h d a \overline{f(g)} \overline{\kappa_{V}\left(a^{-1}\right)} \int d(\widetilde{\Lambda} \phi)(b) \kappa_{V}\left(b^{-1} a^{-1} g^{-1} h\right) f(h) \\
& =\int d(\widetilde{\Lambda} \phi)(b) \int d a \int d g \overline{f(g)} \overline{\kappa_{V}(a g)} \int d h f(h) \kappa_{V}\left(b^{-1} a h\right) \\
& =\int d(\widetilde{\Lambda} \phi)(b) \int d a\left(f * \check{\kappa}_{V}\right)^{*}(a)\left(f * \check{\kappa}_{V}\right)\left(a^{-1} b\right)=\widetilde{\Lambda} \phi\left[\left(f * \kappa_{V}\right)^{*} *\left(f * \kappa_{V}\right)\right],
\end{aligned}
$$

where $\check{\kappa}_{V}(g):=\kappa_{V}\left(g^{-1}\right)=\kappa_{V}(g)$ by property $(i)$ in Eq. (20). Hence, if $\widetilde{\Lambda} \phi$ is a positive definite measure from $M\left(G_{1}\right)$ then for every $U, \Lambda_{U} \phi$ is a positive definite function on $G_{1}$ (note that $f * \kappa_{V}$ is continuous since $f$ is).

Let us introduce some terminology, analogous to that used in the theory of linear mappings of operators on finite-dimensional Hilbert spaces (see e.g. Refs. [32, 19, 18]):

Definition 3.1 Let $\Lambda: C\left(G_{2}\right) \rightarrow C\left(G_{1}\right)$ be a bounded linear map. Then $\Lambda$ is called:

- positive definite $(P D)$ if it preserves positive definite functions, i.e. if $\Lambda \mathcal{P}\left(G_{2}\right) \subset \mathcal{P}\left(G_{1}\right)$;

- $H$-positive definite $(H-P D)$, where $H$ is a compact group, if id $\otimes \Lambda: C\left(H \times G_{2}\right) \rightarrow C(H \times$ $\left.G_{1}\right)$ is positive definite, i.e. if $(i d \otimes \Lambda) \mathcal{P}\left(H \times G_{2}\right) \subset \mathcal{P}\left(H \times G_{1}\right)$;

- completely positive definite (CPD) if it is $H$-positive definite for any compact $H$.

Thus, rephrased in the terms introduced above, Lemma 3.3 states that every bounded linear map $\widetilde{\Lambda}: C\left(G_{2}\right) \rightarrow M\left(G_{1}\right)$, mapping $\mathcal{P}\left(G_{2}\right)$ into positive definite measures, can be weakly-* approximated by positive definite maps from $C\left(G_{2}\right)$ to $C\left(G_{1}\right)$.

After we have established almost all the necessary facts, we return to the main Lemma 3.1 , First, rewrite Eq. (11) using the regularization (3.3) in order to be able to write down explicitly the right hand side of Eq. (11) for arbitrary functions, not only product ones. For product functions we have:

$$
\operatorname{Re} L(f \otimes \xi)=\operatorname{Re} \widetilde{\Lambda}_{L} \xi(f)
$$

We apply the regularization (23) to $\operatorname{Re} \widetilde{\Lambda}_{L}$, denoting the regularized operators by $R_{U}, R_{U}: C\left(G_{2}\right) \rightarrow$ $C\left(G_{1}\right)$, rather than by $\left(\operatorname{Re} \Lambda_{L}\right)_{U}$, to obtain:

$$
\begin{aligned}
\operatorname{Re} L(f \otimes \xi)=\lim _{U \rightarrow\left\{e_{1}\right\}} R_{U} \xi(f) & =\lim _{U \rightarrow\left\{e_{1}\right\}} \int_{G_{1}} d g_{1}\left(R_{U} \xi\right)\left(g_{1}\right) f\left(g_{1}\right) \\
& =\lim _{U \rightarrow\left\{e_{1}\right\}} \int_{G_{1}} d g_{1}\left[\left(\mathrm{id} \otimes R_{U}\right) f \otimes \xi\right]\left(g_{1}, g_{1}\right)
\end{aligned}
$$


Formula (26) immediately extends to all of $C\left(G_{1} \times G_{2}\right)$, so in particular for any $\varphi \in \mathcal{P}_{1}\left(G_{1} \times G_{2}\right)$ we have:

$$
\operatorname{Re} L(\varphi)=\lim _{U \rightarrow\left\{e_{1}\right\}} \int_{G_{1}} d g_{1}\left[\left(\mathrm{id} \otimes R_{U}\right) \varphi\right]\left(g_{1}, g_{1}\right) .
$$

Summarizing, by application of Lemmas 3.2 and 3.3 we obtain that for an arbitrary functional $L \in C\left(G_{1} \times G_{2}\right)^{\prime}$, such that $\operatorname{Re} L\left(\psi_{1} \otimes \psi_{2}\right) \geqslant 0$ for every $\psi_{1}, \psi_{2} \in \mathcal{P}_{1}(G), \operatorname{Re} L(\varphi)$ is given by Eq. (27), where for every neighbourhood $U \subset G_{1}$ the maps $R_{U}: C\left(G_{2}\right) \rightarrow C\left(G_{1}\right)$ are bounded and positive definite. We need two more simple facts.

First, we note that if $\varphi$ is a positive definite function on the product of two copies of $G_{1}$, i.e. $\varphi \in \mathcal{P}\left(G_{1} \times G_{1}\right)$, then its restriction to the diagonal $\left.\varphi\right|_{\Delta}(g):=\varphi(g, g)$ is a positive definite function on $G_{1}$. A particularly direct proof of this fact follows from the GNS construction (cf. Theorem 2.1):

$$
\begin{aligned}
\left.\iint d g d h \overline{f(g)} \varphi\right|_{\Delta}\left(g^{-1} h\right) f(h) & =\iint d g d h \overline{f(g)}\left\langle v_{\varphi} \mid \pi_{\varphi}\left(g^{-1}, g^{-1}\right) \pi_{\varphi}(h, h) v_{\varphi}\right\rangle f(h) \\
& =\left\langle\int d g f(g) \pi_{\varphi}(g, g) v_{\varphi} \mid \int d h f(h) \pi_{\varphi}(h, h) v_{\varphi}\right\rangle \geqslant 0
\end{aligned}
$$

Second, we note that since $G_{1}$ is compact, $\int d g \phi(g) \geqslant 0$ for any $\phi \in \mathcal{P}\left(G_{1}\right)$ (cf. Ref. [27], Theorem 34.8). Indeed, if $G_{1}$ is compact then the constant function 1 is a function with compact support and we can use it in the condition (3), which then implies that $\iint d g d h \phi\left(g^{-1} h\right)=$ $\int d h \phi(h) \geqslant 0$, where we changed variables $h \mapsto g h$ and used the normalization of $d g$.

To finish the proof of the main Theorem 3.2, let us assume that for every bounded and positive definite map $\Lambda: C\left(G_{2}\right) \rightarrow C\left(G_{1}\right)$, the function (id $\left.\otimes \Lambda\right) \varphi$ is positive definite. It then follows from the above discussion and from Eq. (27) that $\operatorname{Re} L(\varphi) \geqslant 0$ for every $L$, such that $\operatorname{Re} L\left(\psi_{1} \otimes \psi_{2}\right) \geqslant 0$ for every $\psi_{1} \in \mathcal{P}_{1}\left(G_{1}\right), \psi_{2} \in \mathcal{P}_{1}\left(G_{2}\right)$. But then Lemma 3.1 implies that $\varphi \in \operatorname{Sep}$.

\section{Fourier transforms and "generating function" formalism}

In this section we establish a connection between the formalism of positive definite functions and standard notions of entanglement theory, thus ascribing a "physical meaning" to the former. Namely, as advertised in the Introduction, we show that various group-theoretical objects studied in the previous sections turn out to be "generating functions" for the corresponding operator-algebraic objects. For example a positive definite function generates a family of (subnormalized) density matrices, a positive definite map (cf. Definition 3.1) generates a family of positive maps, etc. We also derive here a weaker version of the Horodecki Theorem (cf. Theorem 1.2) from Theorem 3.2 and prove a number of other useful results.

Our main tool will be non-commutative Fourier analysis of continuous functions on compact groups. Below we recall some basic notions and methods, which we will need (see e.g. Refs. [25, 26] for more). The goal which we have in mind is to construct uniformly convergent Fourier series for continuous functions.

By (a part of) the fundamental theorem of the theory - the Peter-Weyl Theorem (see e.g. Ref. [25], Theorem 5.12), any continuous function on a compact group can be uniformly approximated by linear combinations of matrix elements of irreducible representations, taken in some fixed orthonormal bases of the corresponding representation spaces. Here, we are 
primarily interested in product groups $G_{1} \times G_{2}$, so we first recall their representation structure. Let us denote by $\widehat{G}_{1}\left(\widehat{G}_{2}\right)$ the set of equivalence classes of irreducible, strongly continuous, unitary representations (irreps) of $G_{1}\left(G_{2}\right)$. Since $G_{1}, G_{2}$ are compact, $\widehat{G}_{1}$ and $\widehat{G}_{2}$ are discrete and (the classes of equivalent) irreducible representations can be labelled by discrete indices. We will denote irreps of $G_{1}$ and $G_{2}$ by $\pi_{\alpha}$ and $\tau_{\beta}$ respectively and the spaces where they act by $\mathcal{H}_{\alpha}$ and $\widetilde{\mathcal{H}}_{\beta}$. It can be then shown that for a large family of groups, including compact ones, every irrep of the product $G_{1} \times G_{2}$ can be chosen in the form $\pi_{\alpha} \otimes \tau_{\beta}$, where:

$$
\pi_{\alpha} \otimes \tau_{\beta}\left(g_{1}, g_{2}\right):=\pi_{\alpha}\left(g_{1}\right) \otimes \tau_{\beta}\left(g_{2}\right)
$$

acts in the space $\mathcal{H}_{\alpha} \otimes \widetilde{\mathcal{H}}_{\beta}$ (cf. Ref. [25], Theorem 7.25; Ref. [26], Proposition 13.1.8). In other words, $\widehat{G_{1} \times G_{2}}$ can be identified with $\widehat{G}_{1} \times \widehat{G}_{2}$.

Next, we need matrix elements of the irreps $\pi_{\alpha} \otimes \tau_{\beta}$. It is natural here to take them with respect to product bases of the representation spaces $\mathcal{H}_{\alpha} \otimes \widetilde{\mathcal{H}}_{\beta}$. Thus, for each pair of the representation indices $\alpha$ and $\beta$ we fix an orthonormal base $\left\{e_{i}\right\}_{i=1, \ldots, d i m \mathcal{H}_{\alpha}}$ of $\mathcal{H}_{\alpha}$ and an orthonormal base $\left\{\tilde{e}_{k}\right\}_{k=1, \ldots, \operatorname{dim} \widetilde{\mathcal{H}}_{\beta}}$ of $\widetilde{\mathcal{H}}_{\beta}$ (we do not indicate explicitly the dependence of $\left\{e_{i}\right\},\left\{\tilde{e}_{k}\right\}$ on the representation indices $\alpha, \beta$ in order not to complicate the notation). The corresponding matrix elements of $\pi_{\alpha} \otimes \tau_{\beta}$ are then simply given by products of the matrix elements of $\pi_{\alpha}$ and $\tau_{\beta}$-that is, they are given by the functions $\pi_{i j}^{\alpha} \otimes \tau_{k l}^{\beta}$, where:

$$
\pi_{i j}^{\alpha}\left(g_{1}\right):=\left\langle e_{i} \mid \pi_{\alpha}\left(g_{1}\right) e_{j}\right\rangle, \quad \tau_{k l}^{\beta}\left(g_{2}\right):=\left\langle\tilde{e}_{k} \mid \tau_{\beta}\left(g_{2}\right) \tilde{e}_{l}\right\rangle .
$$

Now, for a given $f \in C\left(G_{1} \times G_{2}\right)$ we can formally write the Fourier series:

$$
f=\sum_{\alpha, \beta} \sum_{i, \ldots, l} f_{\alpha \beta}^{i j k l} \pi_{i j}^{\alpha} \otimes \tau_{k l}^{\beta}, \quad f_{\alpha \beta}^{i j k l}:=n_{\alpha} m_{\beta} \iint_{G_{1}} d g_{G_{2}} d g_{2} \overline{\pi_{i j}^{\alpha}\left(g_{1}\right)} \overline{\tau_{k l}^{\beta}\left(g_{2}\right)} f\left(g_{1}, g_{2}\right),
$$

where $n_{\alpha}:=\operatorname{dim} \mathcal{H}_{\alpha}, m_{\beta}:=\operatorname{dim} \mathcal{H}_{\beta}$. However, for a generic function $f \in C\left(G_{1} \times G_{2}\right)$ the Fourier series (31) converges only in the $L^{2}$ norm (since $G_{1} \times G_{2}$ is compact $C\left(G_{1} \times G_{2}\right) \subset L^{2}\left(G_{1} \times G_{2}\right)$ ) and not uniformly.

The standard way around this difficulty is the following: i) regularize $f$ so that the Fourier series of the regularized function converges uniformly; ii) perform the desired manipulations with the series; iii) at the end uniformly remove the regularization. For the regularization the same technique as in Section 3 (cf. Eqs. (16,18) and Eqs. (20,-23)) is used. Thus, for a given $f$ we consider a function $f_{\mathcal{U}}:=f * \psi_{\mathcal{U}}$, where the regularizing functions $\psi_{\mathcal{U}} \in C\left(G_{1} \times G_{2}\right)$ are defined in an analogous way as in Eqs. (20+22), but this time on the product $G_{1} \times G_{2}$. The sets $\mathcal{U} \subset G_{1} \times G_{2}$ now run through a neighborhood base of the neutral element $\left\{e_{1}, e_{2}\right\} \in G_{1} \times G_{2}$. Since, by construction, the $\psi_{\mathcal{U}}$ 's are central on $G_{1} \times G_{2}$ (cf. property (ii) in Eq. (20)), a simple calculation shows that the Fourier series of $f * \psi_{\mathcal{U}}$ takes the following form:

$$
f * \psi_{\mathcal{U}}=\sum_{\alpha, \beta} \sum_{i, \ldots, l} c_{\mathcal{U}}^{\alpha \beta} f_{\alpha \beta}^{i j k l} \pi_{i j}^{\alpha} \otimes \tau_{k l}^{\beta}, \quad c_{\mathcal{U}}^{\alpha \beta}:=\iint_{G_{1}} d g_{G_{2}} d g_{2} \psi_{\mathcal{U}}\left(g_{1}, g_{2}\right) \overline{\chi_{\alpha}\left(g_{1}\right)} \overline{\chi_{\beta}\left(g_{2}\right)},
$$

where $\chi_{\alpha}(g):=\operatorname{tr} \pi_{\alpha}(g)$ is the character of the representation $\pi_{\alpha}$ and, analogously, $\chi_{\beta}$ is the character of $\tau_{\beta}$. It can be then shown that the series (32) converges uniformly for every $\mathcal{U}$ (cf. Ref. [25], p. 137). Thus, the role of the constants $c_{\mathcal{U}}^{\alpha \beta}$ is to enhance convergence of the Fourier series (31). Note that since $f * \psi_{\mathcal{U}}=\kappa_{\mathcal{V}}^{*} * f * \kappa_{\mathcal{V}}$, where the sets $\mathcal{V}$ are defined as in Eq. (20) but 
on $G_{1} \times G_{2}$, the regularization preserves positive definiteness (cf. Eq. (24) where we proved it for measures on a single group $G_{1}$ ). In fact, it preserves separability as well, as we will show later (see Lemma 4.1). Finally, the initial function $f$ can be recovered from $f * \psi_{\mathcal{U}}$ by letting $\mathcal{U} \rightarrow\left\{e_{1}, e_{2}\right\}$ as then $f * \psi_{\mathcal{U}} \rightarrow f$ uniformly (cf. Ref. 25], Theorem 2.42).

Let us define operators $\hat{f}_{\alpha \beta} \in \mathcal{L}\left(\mathcal{H}_{\alpha} \otimes \widetilde{\mathcal{H}}_{\beta}\right)$ by:

$$
\hat{f}_{\alpha \beta}:=\sum_{i, \ldots, l} f_{\alpha \beta}^{j i l k}\left|e_{i}\right\rangle\left\langle e_{j}|\otimes| \tilde{e}_{k}\right\rangle\left\langle\tilde{e}_{l}\right|=n_{\alpha} m_{\beta} \iint_{G_{1}} d g_{G_{2}} d g_{2} f\left(g_{1}, g_{2}\right) \pi_{\alpha}\left(g_{1}\right)^{\dagger} \otimes \tau_{\beta}\left(g_{2}\right)^{\dagger},
$$

(note the change of the order of indices). Operators $\hat{f}_{\alpha \beta}$ are inverse Fourier transforms of $f$ : $\hat{f}_{\alpha \beta} \equiv \hat{f}\left(\pi_{\alpha} \otimes \tau_{\beta}\right)$ [25, 20] and Fourier series (31) and (32) can be rewritten as:

$$
f=\sum_{\alpha, \beta} \operatorname{tr}\left[\hat{f}_{\alpha \beta} \pi_{\alpha} \otimes \tau_{\beta}\right], \quad f * \psi_{\mathcal{U}}=\sum_{\alpha, \beta} c_{\mathcal{U}}^{\alpha \beta} \operatorname{tr}\left[\hat{f}_{\alpha \beta} \pi_{\alpha} \otimes \tau_{\beta}\right]
$$

The last equation again explicitly shows the role of regularization in enhancing convergence of the Fourier series (31).

Having recalled the technicalities of the Fourier analysis, we proceed to relate the grouptheoretical formalism to the standard one. We begin by quoting a standard fact, which we will extensively use in what follows (see e.g. Ref. [27], Theorem 34.10):

Theorem 4.1 $\varphi \in \mathcal{P}\left(G_{1} \times G_{2}\right)$ if and only if $\hat{\varphi}_{\alpha \beta} \geqslant 0$ for all $\left[\pi_{\alpha}\right] \in \widehat{G}_{1}$ and $\left[\tau_{\beta}\right] \in \widehat{G}_{2}$.

This is a non-commutative analog of the fact that positive definiteness corresponds under (usual) Fourier transform to positivity.

We present a proof of the above theorem just for completeness' sake. Let us first introduce an abbreviation $\boldsymbol{g}:=\left(g_{1}, g_{2}\right) \in G_{1} \times G_{2}$. From Eq. (33) we then obtain for any $v \in \mathcal{H}_{\alpha} \otimes \widetilde{\mathcal{H}}_{\beta}$ :

$$
\begin{aligned}
\left\langle v \mid \hat{\varphi}_{\alpha \beta} v\right\rangle & =n_{\alpha} m_{\beta} \iint d g_{1} d g_{2} \varphi\left(g_{1}, g_{2}\right)\left\langle v \mid \pi_{\alpha}\left(g_{1}\right)^{\dagger} \otimes \tau_{\beta}\left(g_{2}\right)^{\dagger} v\right\rangle \\
& =n_{\alpha} m_{\beta} \iint d \boldsymbol{h} d \boldsymbol{g} \varphi\left(\boldsymbol{h}^{-1} \boldsymbol{g}\right)\left\langle\pi_{\alpha}\left(h_{1}\right)^{\dagger} \otimes \tau_{\beta}\left(h_{2}\right)^{\dagger} v \mid \pi_{\alpha}\left(g_{1}\right)^{\dagger} \otimes \tau_{\beta}\left(g_{2}\right)^{\dagger} v\right\rangle \\
& \left.=\sum_{i, k=1}^{n_{\alpha}, m_{\beta}} n_{\alpha} m_{\beta} \iint d \boldsymbol{h} d \boldsymbol{g} \overline{v_{i k}^{\alpha \beta}(\boldsymbol{h}}\right) \varphi\left(\boldsymbol{h}^{-1} \boldsymbol{g}\right) v_{i k}^{\alpha \beta}(\boldsymbol{g}) \geqslant 0,
\end{aligned}
$$

where $v_{i k}^{\alpha \beta}(\boldsymbol{h}):=\left\langle e_{i} \otimes \tilde{e}_{k} \mid \pi_{\alpha}\left(h_{1}\right)^{\dagger} \otimes \tau_{\beta}\left(h_{2}\right)^{\dagger} v\right\rangle$. In the second step above we inserted $1=$ $\iint_{G_{1} \times G_{2}} d h_{1} d h_{2}$ and then changed the variables $\boldsymbol{g} \rightarrow \boldsymbol{h}^{-1} \boldsymbol{g}$. Then we inserted the unit matrix $\mathbf{1}_{\alpha} \otimes \mathbf{1}_{\beta}$, decomposed with respect to the fixed bases $\left\{e_{i}\right\},\left\{\tilde{e}_{k}\right\}$ of $\mathcal{H}_{\alpha}, \widetilde{\mathcal{H}}_{\beta}$ and used positive definiteness of $\varphi$. Note that we do not need uniform convergence of the Fourier series here and hence we used the $L^{2}$-convergent series (31) of $\varphi$. The same applies to the proof in the other direction.

Let us now assume that $\hat{\varphi}_{\alpha \beta} \geqslant 0$ for all $\alpha, \beta$. Then from Eq. (31) we obtain:

$$
\begin{aligned}
& \iint d \boldsymbol{g} d \boldsymbol{h} \overline{f(\boldsymbol{g})} \varphi\left(\boldsymbol{g}^{-1} \boldsymbol{h}\right) f(\boldsymbol{h})=\sum_{\alpha, \beta} \sum_{i, \ldots, l} \varphi_{\alpha \beta}^{i j k l} \iint d \boldsymbol{g} d \boldsymbol{h} \overline{f\left(g_{1}, g_{2}\right)} \pi_{i j}^{\alpha}\left(g_{1}^{-1} h_{1}\right) \tau_{k l}^{\beta}\left(g_{2}^{-1} h_{2}\right) f\left(h_{1}, h_{2}\right) \\
& =\sum_{\alpha, \beta} \sum_{i, \ldots, l, r, s} \varphi_{\alpha \beta}^{i j k l} \iint d g_{1} d g_{2} \overline{f\left(g_{1}, g_{2}\right)} \overline{\pi_{r i}^{\alpha}\left(g_{1}\right)} \overline{\tau_{s k}^{\beta}\left(g_{2}\right)} \iint d h_{1} d h_{2} f\left(h_{1}, h_{2}\right) \pi_{r j}^{\alpha}\left(h_{1}\right) \tau_{s l}^{\beta}\left(h_{2}\right) \\
& =\sum_{\alpha, \beta} \sum_{r, s}\left\langle c_{r s} \mid \hat{\varphi}_{\alpha \beta} c_{r s}\right\rangle \geqslant 0
\end{aligned}
$$


for any $f \in C\left(G_{1} \times G_{2}\right)$, where $c_{r s}:=\sum_{i k} \iint d g_{1} d g_{2} f\left(g_{1}, g_{2}\right) \pi_{r i}^{\alpha}\left(g_{1}\right) \tau_{s k}^{\beta}\left(g_{2}\right) e_{i} \otimes \tilde{e}_{k} . \square$

Thus, by the above theorem, a positive definite function generates a family of subnormalized states $\hat{\varphi}_{\alpha \beta}$. The operators $\hat{\varphi}_{\alpha \beta}$ are not normalized (except in the trivial case when sum (34) consists of one term only) even if $\varphi$ is, since from Eq. (34) we obtain that $\sum_{\alpha, \beta} \operatorname{tr}\left(\hat{\varphi}_{\alpha \beta}\right)=$ $\varphi\left(e_{1}, e_{2}\right)=1$, so $\operatorname{tr}\left(\hat{\varphi}_{\alpha \beta}\right) \leqslant 1$. However, we can still speak of separability of the operators $\hat{\varphi}_{\alpha \beta}$ in the sense that they are decomposable into convex combinations of products of positive operators (cf. the corresponding remark after Definition 2.2). The following result holds (cf. Ref. [20] where a weaker version was proven):

Lemma 4.1 A function $\varphi \in \mathcal{P}_{1}\left(G_{1} \times G_{2}\right)$ is separable if and only if the operators $\hat{\varphi}_{\alpha \beta} \in$ $\mathcal{L}\left(\mathcal{H}_{\alpha} \otimes \widetilde{\mathcal{H}}_{\beta}\right)$ are separable for all $\left[\pi_{\alpha}\right] \in \widehat{G}_{1}$ and $\left[\tau_{\beta}\right] \in \widehat{G}_{2}$.

Indeed, from Eq. (33) it follows that if $\operatorname{Sep}_{0} \ni \varphi=\sum_{m=1}^{K} p_{m} \varepsilon_{m} \otimes \eta_{m}$ then $\hat{\varphi}_{\alpha \beta}=$ $\sum_{m=1}^{K} p_{m} \hat{\varepsilon}_{\alpha}^{(m)} \otimes \hat{\eta}_{\beta}^{(m)}$, where all the operators $\hat{\varepsilon}_{\alpha}^{(m)}, \hat{\eta}_{\beta}^{(m)}$ are positive by Lemma 4.1, since $\varepsilon_{m} \in$ $\mathcal{E}_{1}\left(G_{1}\right), \eta_{m} \in \mathcal{E}_{1}\left(G_{2}\right)$ for all $m$. This extends to all of $S e p$ by the continuity for all $\alpha, \beta$ of the inverse Fourier transform (33) $\varphi \mapsto \hat{\varphi}_{\alpha \beta}$ and the fact that positive separable matrices $\sigma$ with $\operatorname{tr} \sigma \leqslant 1$ form a compact convex subset of $\mathcal{L}\left(\mathcal{H}_{\alpha} \otimes \widetilde{\mathcal{H}}_{\beta}\right)$ [16]. The latter follows from the facts that i) the set of extreme points of the latter subset can be identified with $\mathbb{C} P^{n_{\alpha}} \times \mathbb{C} P^{m_{\beta}} \cup\{0\}$ and ii) the convex hull of a compact subset of $\mathbb{R}^{N}$ is compact.

Conversely, assume that for every $\alpha, \beta, \hat{\varphi}_{\alpha \beta}=\sum_{m=1}^{K_{\alpha \beta}} p_{m}^{\alpha \beta}\left|x_{m}^{\alpha}\right\rangle\left\langle x_{m}^{\alpha}|\otimes| y_{m}^{\beta}\right\rangle\left\langle y_{m}^{\beta}\right|$, where $x_{m}^{\alpha} \in$ $\mathcal{H}_{\alpha}, y_{m}^{\beta} \in \widetilde{\mathcal{H}}_{\beta}$. We will prove that $\varphi$ is then a uniform limit of separable functions and hence is itself separable. First, we pass to the regularized function $\varphi_{\mathcal{U}}:=\varphi * \psi_{\mathcal{U}}$, according to the procedure we described above (cf. Eq. (32) and the surrounding paragraph). As we mentioned, $\varphi_{\mathcal{U}}$ is positive definite and hence $\varphi_{\mathcal{U}}\left(e_{1}, e_{2}\right)=\left\|\varphi_{\mathcal{U}}\right\|_{\infty}>0$ (except in the trivial case $\varphi \equiv 0$ ), which allows us to pass to the normalized function $\varphi_{\mathcal{U}} /\left\|\varphi_{\mathcal{U}}\right\|_{\infty}$. Then Eq. (34) implies that:

$$
\begin{aligned}
& \frac{1}{\left\|\varphi_{\mathcal{U}}\right\|_{\infty}} \varphi_{\mathcal{U}}\left(g_{1}, g_{2}\right)=\frac{1}{\left\|\varphi_{\mathcal{U}}\right\|_{\infty}} \sum_{\alpha, \beta} \sum_{m=1}^{K_{\alpha \beta}} c_{\mathcal{U}}^{\alpha \beta} p_{m}^{\alpha \beta}\left\langle x_{m}^{\alpha} \mid \pi_{\alpha}\left(g_{1}\right) x_{m}^{\alpha}\right\rangle\left\langle y_{m}^{\beta} \mid \tau_{\beta}\left(g_{2}\right) y_{m}^{\beta}\right\rangle \\
& =\sum_{\alpha, \beta} \sum_{m=1}^{K_{\alpha \beta}} \frac{1}{\left\|\varphi_{\mathcal{U}}\right\|_{\infty}} c_{\mathcal{U}}^{\alpha \beta} p_{m}^{\alpha \beta}\left\|x_{m}^{\alpha}\right\|^{2}\left\|y_{m}^{\beta}\right\|^{2}\left\langle\frac{x_{m}^{\alpha}}{\left\|x_{m}^{\alpha}\right\|} \mid \pi_{\alpha}\left(g_{1}\right) \frac{x_{m}^{\alpha}}{\left\|x_{m}^{\alpha}\right\|}\right\rangle\left\langle\frac{y_{m}^{\beta}}{\left\|y_{m}^{\beta}\right\|} \mid \tau_{\beta}\left(g_{2}\right) \frac{y_{m}^{\beta}}{\left\|y_{m}^{\beta}\right\|}\right\rangle
\end{aligned}
$$

and the series converges uniformly. The functions given by scalar products belong to $\mathcal{E}_{1}\left(G_{1}\right)$ and $\mathcal{E}_{1}\left(G_{2}\right)$ respectively, since $\pi_{\alpha}$ and $\tau_{\beta}$ are irreducible. From their definition in Eq. (32) and the definition of $\psi_{\mathcal{U}}(22)$ it also follows that the factors $c_{\mathcal{U}}^{\alpha \beta}$ are non-negative:

$$
\begin{aligned}
c_{\mathcal{U}}^{\alpha \beta} & =\iint d \boldsymbol{g} d \boldsymbol{h} \kappa_{\mathcal{V}}(\boldsymbol{h}) \kappa_{\mathcal{V}}\left(\boldsymbol{h}^{-1} \boldsymbol{g}\right) \overline{\chi_{\alpha \beta}(\boldsymbol{g})}=\iint d \boldsymbol{g} d \boldsymbol{h} \kappa_{\mathcal{V}}\left(\boldsymbol{h}^{-1}\right) \kappa_{\mathcal{V}}(\boldsymbol{g}) \overline{\chi_{\alpha \beta}(\boldsymbol{h} \boldsymbol{g})} \\
& =\iint d \boldsymbol{g} d \boldsymbol{h} \kappa_{\mathcal{V}}(\boldsymbol{h}) \kappa_{\mathcal{V}}(\boldsymbol{g}) \overline{\chi_{\alpha \beta}\left(\boldsymbol{h}^{-1} \boldsymbol{g}\right)}=\frac{1}{n_{\alpha}^{2} m_{\beta}^{2}} \overline{\operatorname{tr}\left[\widehat{\kappa \mathcal{V}}_{\alpha \beta}\left(\widehat{\kappa \mathcal{V}}_{\alpha \beta}\right)^{\dagger}\right]} \geqslant 0,
\end{aligned}
$$

where we used definition (33) and the fact that $\kappa_{\mathcal{V}}$ is symmetric (cf. property $(i)$ in Eq. (201)) and real. Evaluating $\varphi_{\mathcal{U}} /\left\|\varphi_{\mathcal{U}}\right\|_{\infty}$ at the neutral element we see that the sum in Eq. (37) is in fact a convex combination of pure product functions (cf. the definition of a pure function in Section 2), since:

$$
\sum_{\alpha, \beta} \sum_{m=1}^{K_{\alpha \beta}} \frac{1}{\left\|\varphi_{\mathcal{U}}\right\|_{\infty}} c_{\mathcal{U}}^{\alpha \beta} p_{m}^{\alpha \beta}\left\|x_{m}^{\alpha}\right\|^{2}\left\|y_{m}^{\beta}\right\|^{2}=\frac{\varphi_{\mathcal{U}}\left(e_{1}, e_{2}\right)}{\left\|\varphi_{\mathcal{U}}\right\|_{\infty}}=1 \text { and } \frac{1}{\left\|\varphi_{\mathcal{U}}\right\|_{\infty}} c_{\mathcal{U}}^{\alpha \beta} p_{m}^{\alpha \beta}\left\|x_{m}^{\alpha}\right\|^{2}\left\|y_{m}^{\beta}\right\|^{2} \geqslant 0 .
$$


Thus, $\varphi_{\mathcal{U}} /\left\|\varphi_{\mathcal{U}}\right\|_{\infty}$ is separable, as a uniform limit of separable functions. Since $\varphi \mathcal{U} \underset{\mathcal{U} \rightarrow\left\{e_{1}, e_{2}\right\}}{\longrightarrow} \varphi$ uniformly, $\varphi \in$ Sep.

From Lemma 4.1 it follows that the problem of describing separable functions on $G_{1} \times G_{2}$ generates a family of separability problems in all pairs of dimensions where $G_{1}$ and $G_{2}$ have irreducible representations. In other words, it plays a role of a "generating function" for this family. Conversely, from the form of the Fourier transformation (2) and its inverse (33) it follows that density matrices on $\mathcal{H}_{\alpha} \otimes \widetilde{\mathcal{H}}_{\beta}$ are in one-to-one correspondence with those functions $\varphi$ from $\mathcal{P}_{1}\left(G_{1} \times G_{2}\right)$, which belong to the (finite-dimensional) linear span of $\pi_{i j}^{\alpha} \otimes \tau_{k l}^{\beta}$, where $\pi_{\alpha}, \tau_{\beta}$ are fixed. Moreover, since for an arbitrary density matrix $\varrho \in \mathcal{L}\left(\mathcal{H}_{\alpha} \otimes \widetilde{\mathcal{H}}_{\beta}\right)$ its Fourier transform $\varphi_{\varrho}$ (cf. Eq. (2)) satisfies:

$$
\left(\widehat{\varphi_{\varrho}}\right)_{\gamma \nu}=\delta_{\alpha \gamma} \delta_{\beta \nu} \varrho
$$

Lemma 4.1 implies that (cf. Ref. [20], Theorem 1):

Corollary 4.1 A state $\varrho$ is separable if and only if $\varphi_{\varrho} \in S e p$.

Next we examine bounded linear maps $\Lambda: C\left(G_{2}\right) \rightarrow C\left(G_{1}\right)$. For an arbitrary function $f \in C\left(G_{2}\right)$, we consider a function $\Lambda f_{U} \in C\left(G_{1}\right)$, where $f_{U}:=f * \psi_{U}$ is the regularization of $f$. Now, the regularizing functions $\psi_{U} \in C\left(G_{2}\right)$ are the single-group functions defined in Eq. (22), but now on the group $G_{2}$, and the sets $U$ run through a neighborhood base of $e_{2} \in G_{2}$. Note, however, that unlike in Section 3 here we are regularizing the argument of $\Lambda$ and not its value. Calculating Fourier transform of $\Lambda f_{U}$ from the single-group version of the definition (33) we obtain:

$$
\begin{aligned}
\left(\widehat{\Lambda f_{U}}\right)_{\alpha} & =n_{\alpha} \int_{G_{1}} d g_{1} \Lambda\left(f * \psi_{U}\right)\left(g_{1}\right) \pi_{\alpha}\left(g_{1}\right)^{\dagger}=n_{\alpha} \int_{G_{1}} d g_{1} \sum_{\beta} c_{U}^{\beta} \sum_{k, l} f_{\beta}^{k l}\left(\Lambda \tau_{k l}^{\beta}\right)\left(g_{1}\right) \pi_{\alpha}\left(g_{1}\right)^{\dagger} \\
& =\sum_{\beta} c_{U}^{\beta} \sum_{k, l} f_{\beta}^{k l} n_{\alpha} \int_{G_{1}} d g_{1}\left(\Lambda \tau_{k l}^{\beta}\right)\left(g_{1}\right) \pi_{\alpha}\left(g_{1}\right)^{\dagger}
\end{aligned}
$$

where we used the uniform convergence of Fourier series for $f_{U}$ and the fact that $\Lambda$ is continuous in the uniform norm. The regularizing constants $c_{U}^{\beta}$ are defined analogously as in Eq. (32), i.e.

$$
c_{U}^{\beta}:=\int_{G_{2}} d g_{2} \psi_{U}\left(g_{2}\right) \overline{\chi_{\beta}\left(g_{2}\right)} .
$$

We will find it useful to define maps $\hat{\Lambda}_{\alpha}^{\beta}: \mathcal{L}\left(\widetilde{\mathcal{H}}_{\beta}\right) \rightarrow \mathcal{L}\left(\mathcal{H}_{\alpha}\right)$ through an analog of Eq. (33):

$$
\hat{\Lambda}_{\alpha}^{\beta}:=\sum_{i, \ldots, l} \Lambda_{\alpha l k}^{j i \beta}\left|E_{i j}\right\rangle_{H S}\left\langle\widetilde{E}_{k l}\right|, \quad \Lambda_{\alpha k l}^{i j \beta}:=n_{\alpha} \int_{G_{1}} d g_{1} \overline{\pi_{i j}^{\alpha}\left(g_{1}\right)}\left(\Lambda \tau_{k l}^{\beta}\right)\left(g_{1}\right)
$$

(note the change of the order of indices), where $\widetilde{E}_{k l}:=\left|\tilde{e}_{k}\right\rangle\left\langle\tilde{e}_{l}\left|, E_{i j}:=\right| e_{i}\right\rangle\left\langle e_{j}\right|$ are the bases of $\mathcal{L}\left(\widetilde{\mathcal{H}}_{\beta}\right)$ and $\mathcal{L}\left(\mathcal{H}_{\alpha}\right)$ respectively, and $\langle A \mid B\rangle_{H S}=\operatorname{tr}\left(A^{\dagger} B\right)$. We can then rewrite Eq. (41) as follows:

$$
\left(\widehat{\Lambda f_{U}}\right)_{\alpha}=\sum_{\beta} c_{U}^{\beta} \hat{\Lambda}_{\alpha}^{\beta} \hat{f}_{\beta}
$$


Note that in the above series all operators $\hat{\Lambda}_{\alpha}^{\beta} \hat{f}_{\beta} \in \mathcal{L}\left(\mathcal{H}_{\alpha}\right)$ are finite-dimensional and hence the convergence can be understood in any of the equivalent norms on $\mathcal{L}\left(\mathcal{H}_{\alpha}\right)$.

Conversely, given an arbitrary map $\Phi: \mathcal{L}\left(\widetilde{\mathcal{H}}_{\beta}\right) \rightarrow \mathcal{L}\left(\mathcal{H}_{\alpha}\right)$ we can Fourier transform it and assign to it a map $\Lambda_{\Phi}: C\left(G_{2}\right) \rightarrow C\left(G_{1}\right)$ through the following formula (compare with Eq. (2) where Fourier transform of operators was defined):

$$
\Lambda_{\Phi} f\left(g_{1}\right):=\operatorname{tr}\left[\left(\Phi \hat{f}_{\beta}\right) \pi_{\alpha}\left(g_{1}\right)\right] \text { for every } f \in C\left(G_{2}\right)
$$

It is obvious that $\Lambda_{\Phi} f \in C\left(G_{1}\right)$, because we consider only continuous representations. Moreover from the definition of $\hat{f}_{\beta}$ we have:

$$
\begin{aligned}
\left\|\Lambda_{\Phi} f\right\|_{\infty} & =m_{\beta} \sup _{g_{1} \in G_{1}}\left|\int_{G_{2}} d g_{2} f\left(g_{2}\right) \operatorname{tr}\left[\left(\Phi \tau_{\beta}^{\dagger}\left(g_{2}\right)\right) \pi_{\alpha}\left(g_{1}\right)\right]\right| \\
& \leqslant m_{\beta}\|f\|_{\infty} \sup _{g_{1} \in G_{1}} \int_{G_{2}} d g_{2}\left|\operatorname{tr}\left[\left(\Phi \tau_{\beta}^{\dagger}\left(g_{2}\right)\right) \pi_{\alpha}\left(g_{1}\right)\right]\right| .
\end{aligned}
$$

The last supremum is finite, as the integrand is continuous and $G$ is compact, and independent of $f$, so that $\Lambda_{\Phi}$ is bounded. The transformation (45) is an inverse of the mapping $\Lambda \mapsto \hat{\Lambda}_{\alpha}^{\beta}$ given by Eq. (43), since by an easy direct calculation one finds that (compare Eq. (40)):

$$
\left(\widehat{\Lambda}_{\Phi}\right)_{\nu}^{\mu}=\delta_{\nu \alpha} \delta_{\mu \beta} \Phi \text {. }
$$

By analogy with Theorem 4.1, which characterizes positive definite functions in terms of their inverse Fourier transforms, one would expect a corresponding characterization of positive definite (PD) maps $\Lambda$ (cf. Definition 3.1) in terms of their inverse Fourier transforms $\hat{\Lambda}_{\alpha}^{\beta}$. The next lemma provides such a characterization:

Lemma 4.2 A bounded linear map $\Lambda: C\left(G_{2}\right) \rightarrow C\left(G_{1}\right)$ is positive definite, i.e. $\Lambda \mathcal{P}\left(G_{2}\right) \subset$ $\mathcal{P}\left(G_{1}\right)$, if and only if the maps $\hat{\Lambda}_{\alpha}^{\beta}: \mathcal{L}\left(\widetilde{\mathcal{H}}_{\beta}\right) \rightarrow \mathcal{L}\left(\mathcal{H}_{\alpha}\right)$ are positive for all $\left[\pi_{\alpha}\right] \in \widehat{G}_{1},\left[\tau_{\beta}\right] \in \widehat{G}_{2}$.

To prove it, let us take an arbitrary $\phi \in \mathcal{P}\left(G_{2}\right)$, which by Theorem 4.1 is equivalent to $\hat{\phi}_{\beta} \geqslant 0$ for all $\beta$. We employ Eq. (44). If all maps $\hat{\Lambda}_{\alpha}^{\beta}$ are positive, then $c_{U}^{\beta} \hat{\Lambda}_{\alpha}^{\beta} \hat{\phi}_{\beta} \geqslant 0$ for all $\beta$, since $c_{U}^{\beta} \geqslant 0$ (cf. Eq. (38) where we proved it for $G_{1} \times G_{2}$ ). Since positive operators form a closed cone in $\mathcal{L}\left(\mathcal{H}_{\alpha}\right)$, the series $\sum_{\beta} c_{U}^{\beta} \hat{\Lambda}_{\alpha}^{\beta} \hat{\phi}_{\beta}$ converges to a positive operator and hence $\left(\widehat{\Lambda \phi_{U}}\right)_{\alpha} \geqslant 0$ for all $\alpha$. Theorem 4.1 implies then that $\Lambda\left(\phi * \psi_{U}\right) \in \mathcal{P}\left(G_{1}\right)$. Taking the limit $U \rightarrow\left\{e_{2}\right\}, \phi * \psi_{U}$ converges to $\phi$ uniformly. Thus, using continuity of $\Lambda$ and uniform closedness of $\mathcal{P}\left(G_{1}\right) \subset C\left(G_{1}\right)$, we obtain that $\Lambda \phi \in \mathcal{P}\left(G_{1}\right)$ for any $\phi \in \mathcal{P}\left(G_{2}\right)$.

Conversely, assume $\Lambda$ to be positive definite. Let us fix $\left[\tau_{\beta}\right] \in \widehat{G}_{2}$ and take an arbitrary positive operator $\varrho \in \mathcal{L}\left(\widetilde{\mathcal{H}}_{\beta}\right)$. Applying Fourier transform (2) to $\varrho$ we obtain its characteristic function:

$$
\phi_{\varrho}\left(g_{2}\right):=\operatorname{tr}\left[\varrho \tau_{\beta}\left(g_{2}\right)\right]=\sum_{k, l} \varrho^{k l} \tau_{l k}^{\beta}\left(g_{2}\right),
$$

which by Theorem 4.1 is positive definite, since $\left(\widehat{\phi}_{\varrho}\right)_{\gamma}=\delta_{\gamma \beta} \varrho \geqslant 0$. Hence, $\Lambda \phi_{\varrho}$ is positive definite too. Then from Eq. (44), where we can neglect the regularization and put $c_{U}^{\beta}=1$ since the Fourier series of $\phi_{\varrho}$ contains only one term, and from Theorem 4.1 we obtain that $\left(\widehat{\Lambda \phi_{\varrho}}\right)_{\alpha}=\hat{\Lambda}_{\alpha}^{\beta} \varrho \geqslant 0$ for all $\alpha$. Since $\tau_{\beta}$ and $\varrho$ were arbitrary, the result follows. $\square$

Thus, from the above lemma and Eq. (47) it follows that (compare Corollary 4.1): 
Corollary 4.2 A map $\Phi: \mathcal{L}\left(\widetilde{\mathcal{H}}_{\beta}\right) \rightarrow \mathcal{L}\left(\mathcal{H}_{\alpha}\right)$ is positive if and only if the map $\Lambda_{\Phi}: C\left(G_{2}\right) \rightarrow$ $C\left(G_{1}\right)$, defined in Eq. 45), is positive definite.

Next we present a characterization of completely positive definite maps (cf. Definition 3.1) in terms of their Fourier transforms. We first prove the following fact:

Lemma 4.3 A bounded linear map $\Lambda: C\left(G_{2}\right) \rightarrow C\left(G_{1}\right)$ is $G_{2}$-positive definite, i.e. (id $\otimes$ $\Lambda) \mathcal{P}\left(G_{2} \times G_{2}\right) \subset \mathcal{P}\left(G_{2} \times G_{1}\right)$, if and only if maps $\hat{\Lambda}_{\alpha}^{\beta}: \mathcal{L}\left(\widetilde{\mathcal{H}}_{\beta}\right) \rightarrow \mathcal{L}\left(\mathcal{H}_{\alpha}\right)$ are completely positive for all $\left[\pi_{\alpha}\right] \in \widehat{G}_{1},\left[\tau_{\beta}\right] \in \widehat{G}_{2}$.

Let $\varphi \in \mathcal{P}\left(G_{2} \times G_{2}\right)$, so by Theorem $4.1 \hat{\varphi}_{\beta \gamma} \geqslant 0$ for all $\left[\tau_{\beta}\right],\left[\tau_{\gamma}\right] \in \widehat{G}_{2}$. Then the obvious generalization of Eq. (44) to $G_{2} \times G_{2}$, implies that:

$$
\left[(\mathrm{id} \widehat{\otimes \Lambda}) \varphi_{\mathcal{U}}\right]_{\beta \alpha}=\sum_{\delta, \gamma} c_{\mathcal{U}}^{\delta \gamma}(\widehat{\operatorname{id} \otimes \Lambda})_{\beta \alpha}^{\delta \gamma} \hat{\varphi}_{\delta \gamma}=\sum_{\gamma} c_{\mathcal{U}}^{\beta \gamma}\left(\mathbf{1}_{\beta} \otimes \hat{\Lambda}_{\alpha}^{\gamma}\right) \hat{\varphi}_{\beta \gamma}
$$

where now $\mathcal{U} \subset G_{2} \times G_{2}$ runs through a neighborhood base of $\left\{e_{2}, e_{2}\right\} \in G_{2} \times G_{2}$. If all maps $\hat{\Lambda}_{\alpha}^{\gamma}$ are completely positive, then $c_{\mathcal{U}}^{\beta \gamma}\left(\mathbf{1}_{\beta} \otimes \hat{\Lambda}_{\alpha}^{\gamma}\right) \hat{\varphi}_{\beta \gamma} \geqslant 0$ as operators from $\mathcal{L}\left(\widetilde{\mathcal{H}}_{\beta} \otimes \widetilde{\mathcal{H}}_{\alpha}\right)$, since $c_{\mathcal{U}}^{\beta \gamma} \geqslant 0$ for all $\beta, \gamma$ (cf. Eq. (38) $)$. From closedness of the cone of positive operators in $\mathcal{L}\left(\widetilde{\mathcal{H}}_{\beta} \otimes \widetilde{\mathcal{H}}_{\alpha}\right)$, the series in Eq. (49) converges to a positive operator as well. Hence $[(\mathrm{id} \widehat{\otimes \Lambda}) \varphi \mathcal{U}]_{\beta \alpha} \geqslant 0$ for all $\alpha, \beta$ and from Theorem 4.1 it follows that $(\mathrm{id} \otimes \Lambda)\left(\varphi * \psi_{\mathcal{U}}\right) \in \mathcal{P}\left(G_{2} \times G_{1}\right)$. We remove the regularization by letting $\mathcal{U} \rightarrow\left\{e_{2}, e_{2}\right\}$ so that $\varphi * \psi_{\mathcal{U}} \rightarrow \varphi$ uniformly. Then from the continuity of id $\otimes \Lambda$ and uniform closedness of $\mathcal{P}\left(G_{2} \times G_{1}\right)$ it follows that (id $\left.\otimes \Lambda\right) \varphi \in \mathcal{P}\left(G_{2} \times G_{1}\right)$ for any $\varphi \in \mathcal{P}\left(G_{2} \times G_{2}\right)$.

For the proof in the other direction, we proceed along the same lines as in the proof of the previous lemma-for arbitrary $\left[\tau_{\beta}\right],\left[\tau_{\gamma}\right] \in \widehat{G}_{2}$ and arbitrary $0 \leqslant \varrho \in \mathcal{L}\left(\widetilde{\mathcal{H}}_{\beta} \otimes \widetilde{\mathcal{H}}_{\gamma}\right)$, we consider the Fourier transform of $\varrho, \varphi_{\varrho}$, defined in Eq. (2). Then positive definiteness of id $\otimes \Lambda$, Theorem 4.1, and Eq.(44) generalized to $G_{2} \times G_{2}$ (with $c_{\mathcal{U}}^{\mu \nu}=1$ as the Fourier series (31) of $\varphi_{\varrho}$ contains only one term) imply that $\left[(\mathrm{id} \widehat{\otimes \Lambda}) \varphi_{\varrho}\right]_{\beta \alpha}=\left(\mathbf{1}_{\beta} \otimes \hat{\Lambda}_{\alpha}^{\gamma}\right) \varrho \geqslant 0$ for all $\gamma$. Since $\left[\tau_{\beta}\right],\left[\tau_{\gamma}\right]$, and $\varrho$ are arbitrary, $G_{2}$-positive definiteness of $\Lambda$ implies that every map $\hat{\Lambda}_{\alpha}^{\gamma}$ is $(\operatorname{dim} \tau)$-positive for all possible $[\tau] \in \widehat{G}_{2}$. Thus, in particular, every $\hat{\Lambda}_{\alpha}^{\gamma}$ is $m_{\gamma}$-positive, $m_{\gamma}=\operatorname{dim} \widetilde{\mathcal{H}}_{\gamma}$. But then by the Choi Theorem (cf. Ref. 18, Theorem 2) this is equivalent to $\hat{\Lambda}_{\alpha}^{\gamma}$ being completely positive.

As a by-product we obtain an analog of the Choi Theorem (cf. Ref. [18], Theorem 2) for PD maps:

Corollary 4.3 A map $\Lambda: C\left(G_{2}\right) \rightarrow C\left(G_{1}\right)$ is completely positive definite if and only if it is $G_{2}$-positive definite.

The proof in one direction follows immediately from Definition 3.1. For the opposite implication, let us assume that $\Lambda$ is $G_{2}$-PD. Let $H$ be an arbitrary compact group and let Latin indices $a, b, \ldots$ enumerate irreps of $H$. From the complete positivity of Fourier transforms $\hat{\Lambda}_{\alpha}^{\beta}$, guaranteed by Lemma 4.3, Theorem 4.1, Eq. (38) applied to $H \times G_{2}$, and closedness of the cone of positive operators, we obtain that:

$$
\left[(\mathrm{id} \widehat{\otimes \Lambda}) \varphi_{\mathcal{U}}\right]_{b \alpha}=\sum_{\beta} c_{\mathcal{U}}^{b \beta}\left(\mathbf{1}_{b} \otimes \hat{\Lambda}_{\alpha}^{\beta}\right) \hat{\varphi}_{b \beta} \geqslant 0
$$


for every $b$ and $\alpha$ and every $\varphi \in \mathcal{P}\left(H \times G_{2}\right)$. Thus from Theorem 4.1, (id $\left.\otimes \Lambda\right) \varphi_{\mathcal{U}} \in \mathcal{P}\left(H \times G_{1}\right)$. Letting $\mathcal{U} \rightarrow\left\{e_{H}, e_{2}\right\} \in H \times G_{2}$, so that $\varphi_{\mathcal{U}}$ converges to $\varphi$ uniformly, and using continuity of id $\otimes \Lambda$ and uniform closedness of $\mathcal{P}\left(H \times G_{1}\right)$, we obtain that (id $\left.\otimes \Lambda\right) \varphi \in \mathcal{P}\left(H \times G_{1}\right)$ for every compact $H$ and every $\varphi \in \mathcal{P}\left(H \times G_{2}\right)$.

From Lemma 4.3 and Corollary 4.3 we finally obtain:

Lemma 4.4 A bounded linear map $\Lambda: C\left(G_{2}\right) \rightarrow C\left(G_{1}\right)$ is completely positive definite if and only if the maps $\hat{\Lambda}_{\alpha}^{\beta}: \mathcal{L}\left(\widetilde{\mathcal{H}}_{\beta}\right) \rightarrow \mathcal{L}\left(\mathcal{H}_{\alpha}\right)$ are completely positive for all $\left[\pi_{\alpha}\right] \in \widehat{G}_{1},\left[\tau_{\beta}\right] \in \widehat{G}_{2}$.

Comparison of Theorem 3.2 with the Horodecki Theorem 1.2 shows that positive definite mappings of continuous functions on compact groups play an analogous role to that of positive mappings of density matrices in the standard theory of entanglement [14]. The harmonicanalytical formalism described above makes this observation, as well as the "generating function" analogy, formal. Namely, Lemma 4.2 implies that every PD map $\Lambda$ generates a family of positive maps $\hat{\Lambda}_{\alpha}^{\beta}$, acting between algebras of operators on representation spaces of $G_{1}$ and $G_{2}$. Conversely, Fourier transform (45), together with property (47) and Lemma 4.2 allows one to assign a unique PD map to every suitable (cf. definition (45)) positive map. Analogously, Lemma 4.4 shows that each CPD map gives rise to a family of completely positive maps, and by Fourier transform (45) every (suitable) completely positive map defines a CPD map. Thus, PD and CPD maps between groups play a role of "generating functions" of families of positive and completely positive maps respectively.

In order to compare Theorem 3.2 with the Horodecki Theorem 1.2, we first choose $G_{1}$ and $G_{2}$ so that they possess irreps in dimensions $\operatorname{dim} \mathcal{H}_{\mathscr{A}}$ and $\operatorname{dim} \mathcal{H}_{\mathscr{B}}$, so that we may identify $\mathcal{H}_{\mathscr{A}} \cong \mathcal{H}_{\alpha}$ and $\mathcal{H}_{\mathscr{B}} \cong \widetilde{\mathcal{H}}_{\beta}$ for some $\left[\pi_{\alpha}\right] \in \widehat{G}_{1}$ and $\left[\tau_{\beta}\right] \in \widehat{G}_{2}[20$. Apart from that there are no further restrictions on $G_{1}, G_{2}$. Finding such a group for a given finite-dimensional $\mathcal{H}_{\mathscr{A}}, \mathcal{H}_{\mathscr{B}}$ is always possible - for example we can take $G_{1}=G_{2}=S U(2)$, which possesses irreps in all finite dimensions. Having made the above identification, we obtain from Theorem 3.2 that:

Theorem 4.2A density matrix $\varrho \in \mathcal{L}\left(\mathcal{H}_{\alpha} \otimes \widetilde{\mathcal{H}}_{\beta}\right)$ is separable if and only if for all $\left[\pi_{\gamma}\right] \in \widehat{G}_{1}$ and all positive maps $\Phi_{\gamma}: \mathcal{L}\left(\widetilde{\mathcal{H}}_{\beta}\right) \rightarrow \mathcal{L}\left(\mathcal{H}_{\gamma}\right),\left(\mathbf{1}_{\alpha} \otimes \Phi_{\gamma}\right) \varrho \geqslant 0$ as an operator on $\mathcal{H}_{\alpha} \otimes \mathcal{H}_{\gamma}$.

To prove it, we first Fourier transform $\varrho$, passing to its characteristic function $\varphi_{\varrho}=\operatorname{tr}\left(\varrho \pi_{\alpha} \otimes\right.$ $\left.\tau_{\beta}\right) \in \mathcal{P}_{1}\left(G_{1} \times G_{2}\right)$. From Lemma $4.1 \varrho$ is separable if and only if $\varphi \in S e p$. Applying Theorem 3.2 to $\varphi_{\varrho}$ we obtain that $\varrho$ is separable if and only if for all positive definite maps $\Lambda: C\left(G_{2}\right) \rightarrow$ $C\left(G_{1}\right)$, (id $\left.\otimes \Lambda\right) \varphi_{\varrho} \in \mathcal{P}\left(G_{1} \times G_{1}\right)$. From the generalization of Eq. (44) to $G_{1} \times G_{2}$ with all $c_{\mathcal{U}}^{\delta \gamma}=1$ (no regularization of $\varphi_{\varrho}$ is needed because the Fourier series of $\varphi_{\varrho}$ contains only one non-zero term; cf. Eq. (40)) we obtain that:

$$
\left[(\mathrm{id} \widehat{\otimes \Lambda}) \varphi_{\varrho}\right]_{\alpha \gamma}=\left(\mathbf{1}_{\alpha} \otimes \hat{\Lambda}_{\gamma}^{\beta}\right) \varrho .
$$

Then from Theorem 4.1 and Lemma 4.2 it follows that $(\mathrm{id} \otimes \Lambda) \varphi_{\varrho}$ is a positive definite function if and only if $\left(\mathbf{1}_{\alpha} \otimes \hat{\Lambda}_{\gamma}^{\beta}\right) \varrho \geqslant 0$ for every $\gamma$, where every map $\hat{\Lambda}_{\gamma}^{\beta}: \mathcal{L}\left(\widetilde{\mathcal{H}}_{\beta}\right) \rightarrow \mathcal{L}\left(\mathcal{H}_{\gamma}\right)$ is positive. $\square$

Thus, when applied to finite dimension, Theorem 3.2 turns out to be weaker then Theorem 1.2, since generically one has to check positive maps operating between the fixed space $\mathcal{L}\left(\widetilde{\mathcal{H}}_{\beta}\right)$ and the whole family of spaces $\mathcal{L}\left(\mathcal{H}_{\gamma}\right),\left[\pi_{\gamma}\right] \in \widehat{G}_{1}$, and not only between $\mathcal{L}\left(\widetilde{\mathcal{H}}_{\beta}\right)$ and $\mathcal{L}\left(\mathcal{H}_{\alpha}\right)$ as in Theorem 1.2. Note, however, that the number of spaces $\mathcal{H}_{\gamma}$ to check need not be infinite, since it may be possible to find discrete $G_{1}$ [20]. This is particularly easy for low-dimensional initial spaces $\mathcal{H}_{\mathscr{A}}, \mathcal{H}_{\mathscr{B}}$. 


\section{$5 \quad$ Examples of positive definite maps for $G_{1}=G_{2}$}

From the point of view of classification of separable functions using Theorem 3.2 only those positive definite maps which are not completely positive definite (cf. Definition 3.1) are interesting: if $\Lambda$ is a CPD map then all the functions (id $\otimes \Lambda) \varphi, \varphi \in \mathcal{P}_{1}\left(G_{1} \times G_{2}\right)$, are positive definite, whether $\varphi$ is separable or not. Hence we encounter a similar problem as in the finitedimensional linear algebra [19]: classify all positive definite but not completely positive definite maps from $C\left(G_{2}\right)$ to $C\left(G_{1}\right)$. In this Section we give some examples of PD and CPD maps for the case $G_{1}=G_{2} \equiv G$.

The first example of PD but not CPD map was already encountered in Theorem 3.1 - the inversion map $\theta$ :

$$
\theta f(g):=f\left(g^{-1}\right)
$$

To show that $\theta$ is not CPD (positive definiteness will be proven below in a more general setting), observe that $\theta$ corresponds through the Fourier transform to the transposition map $T, T \varrho:=\varrho^{T}$, acting on each representation space $\mathcal{H}_{\alpha}$ of $G$ :

$$
f\left(g^{-1}\right)=\sum_{\alpha} \sum_{i, j} f_{\alpha}^{i j} \pi_{i j}^{\alpha}\left(g^{-1}\right)=\sum_{\alpha} \sum_{i, j} f_{\alpha}^{j i} \overline{\pi_{i j}^{\alpha}(g)}, \quad \text { and hence } \quad(\widehat{\theta f})_{\bar{\alpha}}=T \hat{f}_{\alpha}=\hat{f}_{\alpha}^{T} .
$$

Here index $\bar{\alpha}$ denotes the complex conjugate $\bar{\pi}_{\alpha}$ of representation $\pi_{\alpha}: \bar{\pi}_{\alpha}(g):=\overline{\pi_{\alpha}(g)}$. Eq. (53) establishes the connection between the PPT criterion (Theorem 1.1) and Theorem 3.1 (see Ref. [20] for more details). Now, let $\varrho \in \mathcal{L}\left(\mathcal{H}_{\alpha} \otimes \mathcal{H}_{\beta}\right)$ be any positive operator such that its partial transpose $\left(\mathbf{1}_{\alpha} \otimes T\right) \varrho$ is not positive. Then, by Theorem 4.1 , the Fourier transform $\varphi_{\varrho}$ of $\varrho$ is positive definite, but $(\mathrm{id} \otimes \theta) \varphi_{\varrho}$ is not. We propose to use the same terminology as in quantum information theory and call entangled functions $\varphi$ not detected by $\theta$ (see the remark after Theorem [3.2) bound entangled.

Let us consider more general substitutions of the argument in the tested function. Let $\boldsymbol{\alpha}$ be an arbitrary automorphism of $G$ and $\boldsymbol{\beta}$ an arbitrary anti-automorphism of $G$, i.e.:

$$
\begin{aligned}
\boldsymbol{\alpha}(g h) & =\boldsymbol{\alpha}(g) \boldsymbol{\alpha}(h), \\
\boldsymbol{\beta}(g h) & =\boldsymbol{\beta}(h) \boldsymbol{\beta}(g) .
\end{aligned}
$$

We define the corresponding maps from $C(G)$ to $C(G)$ :

$$
\Lambda_{\boldsymbol{\alpha}} f(g):=f(\boldsymbol{\alpha}(g)), \quad \Lambda_{\boldsymbol{\beta}} f(g):=f(\boldsymbol{\beta}(g)) .
$$

Both $\Lambda_{\boldsymbol{\alpha}}$ and $\Lambda_{\boldsymbol{\beta}}$ are positive definite, which follows most directly from the GNS construction (cf. Theorem 2.1) :

$$
\begin{aligned}
\iint d g d h \overline{f(g)}\left(\Lambda_{\boldsymbol{\alpha}} \phi\right)\left(g^{-1} h\right) f(h) & =\left\langle\int d g f(g) \pi_{\phi}(\boldsymbol{\alpha}(g)) v_{\phi} \mid \int d h f(h) \pi_{\phi}(\boldsymbol{\alpha}(h)) v_{\phi}\right\rangle \geqslant 0, \\
\iint d g d h \overline{f(g)}\left(\Lambda_{\boldsymbol{\beta}} \phi\right)\left(g^{-1} h\right) f(h) & =\left\langle\int d h \overline{f(h)} \pi_{\phi}(\boldsymbol{\beta}(h))^{\dagger} v_{\phi} \mid \int d g \overline{f(g)} \pi_{\phi}(\boldsymbol{\beta}(h))^{\dagger} v_{\phi}\right\rangle \geqslant 0,
\end{aligned}
$$

where $\phi \in \mathcal{P}(G)$ and we used the fact that $\boldsymbol{\alpha}\left(g^{-1}\right)=\boldsymbol{\alpha}(g)^{-1}$ and $\boldsymbol{\beta}\left(g^{-1}\right)=\boldsymbol{\beta}(g)^{-1}$. Moreover, maps arising from automorphisms are completely positive definite. Indeed, from Corollary 4.3 
it is enough to check the extension of $\Lambda$ to $C(G \times G)$. But then we obtain that (with the boldface characters denoting elements of $G \times G)$ :

$$
\begin{aligned}
& \iint d \boldsymbol{g} d \boldsymbol{h} \overline{f(\boldsymbol{g})}\left(\mathrm{id} \otimes \Lambda_{\boldsymbol{\alpha}} \varphi\right)\left(\boldsymbol{g}^{-1} \boldsymbol{h}\right) f(\boldsymbol{h})= \\
& \left\langle\iint d g_{1} d g_{2} f\left(g_{1}, g_{2}\right) \pi_{\varphi}\left(g_{1}, \boldsymbol{\alpha}\left(g_{2}\right)\right) v_{\varphi} \mid \iint d h_{1} d h_{2} f\left(h_{1}, h_{2}\right) \pi_{\varphi}\left(h_{1}, \boldsymbol{\alpha}\left(h_{2}\right)\right) v_{\varphi}\right\rangle \geqslant 0,
\end{aligned}
$$

for an arbitrary $f \in C(G \times G)$. The maps arising from anti-automorphisms are not necessarily CPD - the above calculation leading to the inequality (57) cannot be repeated. However, since every anti-automorphism can be written in the form $\boldsymbol{\beta}(g)=[\boldsymbol{\alpha}(g)]^{-1}$, where $\boldsymbol{\alpha}$ is an automorphism, every map $\Lambda_{\boldsymbol{\beta}}$ arising from an anti-automorphism is of the form:

$$
\Lambda_{\boldsymbol{\beta}}=\Lambda^{C P D} \circ \theta
$$

where $\Lambda^{C P D}$ is some CPD map. Hence, the use of a general anti-homomorphism $\boldsymbol{\beta}$ in Theorem 3.1 gives no improvement, since the function $\left(\mathrm{id} \otimes \Lambda_{\boldsymbol{\beta}}\right) \varphi$ is positive definite if $(\mathrm{id} \otimes \theta) \varphi$ is. In other words, PD maps of the type (58) cannot detect bound entangled functions. This is in close analogy to what one encounters in the study of standard separability problems. Indeed, from Lemma 4.3, Corollary 4.3, and Eq. (53) PD maps of the form (58) generate positive maps of the type $\Phi^{C P} \circ T$, where $\Phi^{C P}$ is a completely positive map. Clearly, by Theorem 1.2, such maps cannot detect bound entangled states $\varrho$, for which $(1 \otimes T) \varrho \geqslant 0$.

We can give a more general example of a CPD map, motivated by the Kraus decomposition of a completely positive map (cf. Ref. [32] and Ref. [18], Theorem 1). For an arbitrary measure $\mu$ from $M(G)$ we define a map:

$$
\Lambda_{\mu} f(g):=\left(\mu^{*} * f * \mu\right)(g)=\iint_{G} \overline{d \mu(a)} d \mu(b) f\left(a g b^{-1}\right),
$$

where the adjoint $\mu^{*}$ is defined as $\mu^{*}(\Omega):=\overline{\mu\left(\Omega^{-1}\right)}$ for any Borel set $\Omega \subset G$ (cf. the corresponding definition for functions after Eq. (13)) and the convolution is defined through Eq. (15). Obviously, $\Lambda_{\mu}$ maps $C(G)$ to $C(G)$ and is a generalization of the regularization formula (23). The map $\Lambda_{\mu}$ is bounded on $C(G)$, since $\sup _{g \in G} \sup _{\|f\|_{\infty}=1}\left|\iint d \overline{\mu(a)} d \mu(b) f\left(a g b^{-1}\right)\right|=|\mu|^{2}(G)$. It is also completely positive definite, which can be easily proven using the GNS Theorem 2.1.

$$
\begin{aligned}
\left(\mathrm{id} \otimes \Lambda_{\mu}\right) \varphi\left(g_{1}, g_{2}\right) & =\iint \overline{d \mu(a)} d \mu(b)\left\langle v_{\varphi} \mid \pi_{\varphi}\left(g_{1}, a g_{2} b^{-1}\right) v_{\varphi}\right\rangle \\
& =\iint \overline{d \mu(a)} d \mu(b)\left\langle\pi_{\varphi}(e, a)^{\dagger} v_{\varphi} \mid \pi_{\varphi}\left(g_{1}, g_{2}\right) \pi_{\varphi}(e, b)^{\dagger} v_{\varphi}\right\rangle \\
& =\left\langle\pi_{\varphi}(e, \mu)^{\dagger} v_{\varphi} \mid \pi_{\varphi}\left(g_{1}, g_{2}\right) \pi_{\varphi}(e, \mu)^{\dagger} v_{\varphi}\right\rangle,
\end{aligned}
$$

where $\pi_{\varphi}(e, \mu):=\int d \mu(g) \pi_{\varphi}(e, g)$. Thus, $\left(\mathrm{id} \otimes \Lambda_{\mu}\right) \varphi$ is positive definite.

Map (59) can be further generalized:

$$
\Lambda_{\mathfrak{M}} f(g):=\int_{M(G)} d \mathfrak{M}(\mu)\left(\mu^{*} * f * \mu\right)(g)
$$

where $\mathfrak{M}$ is a positive measure on $M(G)$ with a finite total variation, i.e. $\mathfrak{M} \in M(M(G))$. We conjecture that any CPD map from $C(G)$ to $C(G)$ is of this form for some measure $\mathfrak{M}$, so that Eq. (61) is an analog of the Kraus decomposition of a completely positive map. 


\section{Conclusions}

The main conclusions are twofold. On one hand, this paper is directed to the audience of mathematicians working in the area of harmonic analysis. We have formulated here the separability problem in purely abstract terms of positive definite functions on compact groups. To our knowledge this is a new problem in harmonic analysis. One may hope that some well established methods of harmonic analysis will help to get more insight into the problem, solving it, at least partially. Several generalizations call for immediate attention: applications to quantum groups being perhaps one of the most fascinating ones. We hope that studies of entanglement within the harmonic analysis framework will open new avenues here.

One the other hand, we expect that the harmonic analysis methods will help to study the physics of separability and entanglement. We expect to find new entanglement criteria, and get a better understanding of the whole problem, by looking at concrete examples and applications of our theoretical results. In particular, finite groups (which have finitely many irreducible representations) of various types (nilpotent, solvable) seem a rich source of interesting examples.

As seen above, passing from linear-algebraic entanglement criteria for quantum states to the theory of positive-definite functions on compact groups is somewhat inovolved and requires technical work. We expect, on the other hand, that, going in the opposite direction, the above general results, when specialized to concrete groups (e.g. finite groups or $S U(2)$ ) will directly yield physically interesting results.

\section{Acknowledgements}

We would like to thank M. Bożejko, P. Horodecki, M. Marciniak, P. Sołtan, and S. L. Woronowicz for discussions. We gratefully acknowledge the financial support of EU IP Programme "SCALA", ESF PESC Programme "QUDEDIS", Spanish MEC grants (FIS 2005-04627, Conslider Ingenio 2010 "QOIT"), and Trup Cualitat Generalitat de Catalunya. JW was partially supported by the NSF grant DMS 9706915.

\section{References}

[1] J. S. Bell, Speakable and Unspeakable in Quantum Mechamics, (Cambridge University Press, Cambridge, 2004).

[2] T. Norsen, Found. Phys. Lett. 19, 633 (2006); Found. Phys. 37, 311 (2007).

[3] A. Peres, Quantum Theory: Concepts and Mehtods, (Kluwer Academic Publishers, Dordrecht, 1993).

[4] R. Horodecki, P. Horodecki, M. Horodecki, and K. Horodecki, Rev. Mod. Phys., in press; arXiv:quant-ph/0702225v2.

[5] E. Strømer, Acta Math. 110, 233 (1963).

[6] B. M. Terhal, Lin. Alg. Appl. 323, 61 (2001); M. Lewenstein, B. Kraus, P. Horodecki, and J. I. Cirac, Phys. Rev. A 63, 044304 (2001); H.-P. Breuer, Phys. Rev. Lett. 97, 080501 (2006).

[7] M. A. Nielsen and I. L. Chuang, Quantum Computation and Quantum Information (Cambridge University Press, Cambridge, 2000). 
[8] A. Einstein, B. Podolsky, and N. Rosen, Phys. Rev. 47, 777 (1935).

[9] R. F. Werner, Phys. Rev. A 40, 4277 (1989).

[10] A. C. Doherty, P. A. Parrilo, and F. M. Spedalieri, Phys. Rev. Lett. 88, 187904 (2002).

[11] H. J. Woerdmann, Phys. Rev. A 67, 010303 (2003).

[12] L. Gurvits, J. Comp. Sys. Sci. 69, 448 (2004).

[13] A. Peres, Phys. Rev. Lett. 77, 1413 (1996).

[14] M. Horodecki, P. Horodecki, and R. Horodecki, Phys. Lett. A 223, 1 (1996).

[15] M.-D. Choi, Linear Algebra Appl. 12, 95 (1975).

[16] P. Horodecki, Phys. Lett. A 232, 333 (1997).

[17] G. Giedke, B. Kraus, M. Lewenstein, and J. I. Cirac, Phys. Rev. Lett. 87, 167904 (2001).

[18] M.-D. Choi, Linear Algebra Appl. 10, 285 (1975).

[19] S. L. Woronowicz, Rep. Math. Phys. 10, 165 (1976); Comm. Math. Phys. 51, 243 (1976);

P. Kruszyński and S. L. Woronowicz, Lett. Math. Phys. 3, 317 (1979).

[20] J. K. Korbicz and M. Lewenstein, Phys. Rev. A 74, 022318 (2006).

[21] J. K. Korbicz and M. Lewenstein, Found. Phys. 37, 879 (2007).

[22] A. S. Holevo, Probabilistic and statistical aspects of quantum theory (North Holland, Amsterdam, 1982).

[23] A. Perelomov, Generalized Coherent States and Their Applications (Springer, Berlin, 1986).

[24] H. Barnum, E. Knill, G. Ortiz, and L. Viola, Phys. Rev. A 68, 032308 (2003); H. Barnum, E. Knill, G. Ortiz, R. Somma, and L. Viola, Phys. Rev. Lett. 92, 107902 (2004); S. L. Braunstein, C. M. Caves, R. Jozsa, N. Linden, S. Popescu, and R. Schack, Phys. Rev. Lett 83, 1054 (1999); F. Mintert and K. Życzkowski, Phys. Rev. A 69, 022317 (2004).

[25] G. Folland, A Course in Abstract Harmonic Analysis (CRC Press, Boca Raton, 1995).

[26] J. Dixmier, $C^{*}$-Algebras, (North Holland, Amsterdam, 1977).

[27] E. Hewitt and K. A. Ross, Abstract Harmonic Analysis, Vol. II (Springer-Verlag, Berlin, 1963).

[28] G. Folland, Real Analysis. Modern Techniques and Their Applications, (Wiley, New York, 1999).

[29] M. Reed and B. Simon, Methods of Modern Mathematical Physics, Vol. I (Academic Press, San Diego, 1980).

[30] A. Jamiołkowski, Rep. Math. Phys. 3, 275 (1972).

[31] R. Godement, Trans. Amer. Math. Soc. 63, 1 (1948).

[32] K. Kraus, States, Effects, and Operators: Fundamental Notions of Quantum Theory (Springer, Berlin, 1983). 